\title{
Electrochemical performance of zinc carbodiimides based porous nanocomposites as supercapacitors
}

\author{
Jiaqi Shen $^{\mathrm{a}, 1}$, Xiaozhen Chen ${ }^{\mathrm{a}, 1}$, Peng Wang ${ }^{\mathrm{a}, \mathrm{b}, *}$, Feng Zhou ${ }^{\mathrm{c}, *}$, Lei Lu ${ }^{\mathrm{a}}$, Rongfang Wang ${ }^{\mathrm{d}}$, \\ Vladimir Linkov ${ }^{\mathrm{e}}$, Shan $\mathrm{Ji}^{\mathrm{a}}{ }^{\mathrm{a}}$ * \\ ${ }^{a}$ College of Biological, Chemical Science and Chemical Engineering, Jiaxing University, Jiaxing 314001, China \\ b Department of Chemistry, University of Illinois at Chicago, 845 W Taylor St., Science and Engineering South (MC 111), Chicago, IL 60607, USA \\ ${ }^{\mathrm{c}}$ College of Chemistry, Chemical Engineering and Materials Science, Collaborative Innovation Center of Suzhou Nano Science and Technology, Soochow University, \\ Suzhou, Jiangsu 215123, China \\ ${ }^{\mathrm{d}}$ College of Chemical Engineering, Qingdao University of Science and Technology, Qingdao 266042, China \\ ${ }^{\mathrm{e}}$ South African Institute for Advanced Materials Chemistry, University of the Western Cape, Cape Town 7535, South Africa
}

\section{A R T I C L E I N F O}

\section{Keywords:}

Energy storage

Supercapacitor

ZIF-8

Graphitic carbon nitride

Zinc carbodiimides

\begin{abstract}
A B S T R A C T
The low energy densities of supercapacitors are generally limited by the used anodes. To develop supercapacitors with high energy densities, metal-organic framework (TRD-ZIF-8) derived honeycomb-like porous zinc carbodiimide (ZnNCN) based nanocomposites (HPZC) surface loaded with graphitized carbon nitride $\left(\mathrm{g}-\mathrm{C}_{3} \mathrm{~N}_{4}\right)$ are synthesized. After their detailed characterization by means of electron microscopy, spectroscopy and electrochemical techniques, the materials are used to prepare asymmetric supercapacitor cells (HPZC-4//AC ASCs) with HPZC-4 and active carbon as electrodes, which demonstrate power and energy densities as high as $7839 \mathrm{~W} \mathrm{Kg}$ and $213 \mathrm{Wh} \mathrm{Kg}^{-1}$, respectively. The unique honeycomb-like porous structure of HPZC loaded with a 2D material $\left(\mathrm{g}-\mathrm{C}_{3} \mathrm{~N}_{4}\right)$ improves charge/mass transport efficiency and reduces ion diffusion resistance, contributing to a specific capacitance of $779 \mathrm{~F} \mathrm{~g}^{-1}$ at a current density of $3 \mathrm{~A} \mathrm{~g}^{-1}$. The study thus presents a new way to design transition metal carbodiimide materials and assesses their potential application as electrodes in asymmetric supercapacitors.
\end{abstract}

\section{Introduction}

Supercapacitors are a new generation of energy storage equipment with advantages such as high power density $\left(>10,000 \mathrm{~W} \mathrm{~kg}^{-1}\right)$, long cycle life $\left(>10^{5}\right.$ cycles), low maintenance costs, and environmental benignity [1,2]. Among supercapacitor types, pseudocapacitances show great potential due to their high energy density and fast reversible surface redox reactions $[3,4]$. Currently, transition metal oxides (TMOs) of $\mathrm{Fe}, \mathrm{Ni}, \mathrm{Co}, \mathrm{Cu}, \mathrm{Zn}$, etc, are most widely used electrode materials in pseudocapacitors due their low cost and high availability [5]. However, TMO based materials generally have poor electrical conductivity, rapid capacity decay and poor cycle stability, which severely restrict their electrochemical applicability [6-8]. At present, those drawbacks are mainly solved by using TMOs in the form of small particles or core-shell and hollow nanostructures $[9,10]$, however, their expensive and lowyield complex synthesis methods offset inherent advantages of these materials, such as their low cost.

Pseudocapacitor electrode cost reduction can be achieved by the use of transition metal carbodiimides (TMNCNs), which possess structures similar to corresponding TMOs [11]. Among these materials, zinc carbodiimide ( $\mathrm{nnNCN}$ ) demonstrated higher theoretical capacity, better cycling life and rate capability than those of the corresponding oxide $(\mathrm{ZnO})$, and does not require complicated processing such as nano-scale tailoring or application of coatings [12-14]. Unfortunately, ZnNCN generally requires unconventional synthetic routes that lead to larger crystal sizes and lower surface areas. Considering the dependence of nanomaterial properties on physical dimensions of their structural units, nanoscale ZnNCN with a high surface area can be expected to demonstrate improved electrochemical performance in energy storage applications, possibly in combination with stable carbon materials for cost reduction.

Generally, high surface area porous carbon materials are considered

\footnotetext{
* Corresponding authors at: College of Biological, Chemical Science and Chemical Engineering, Jiaxing University, Jiaxing, 314001 China (P. Wang).

E-mail addresses: pengwang@zjxu.edu.cn (P. Wang), zhoufeng20031341@suda.edu.cn (F. Zhou), jishan@zjxu.edu.cn (S. Ji).

1 These authors contributed equally to this work.
} 
the ideal choice [15]. However, due to their poor conductivity and uneven pore size distribution, numerous reported porous carbon-based electrodes show insufficient specific capacitance or poor rate capability, leading to the unsatisfactory long cycle performance $[16,17]$. To address these problems, some porous carbon materials with special nanostructures (i.e., core-shell structure and hierarchically structure, etc.) have been designed $[18,19]$. For example, Lei et al. designed a selfsupporting carbon-coated TiN nanotube array, which can improve durable ability remarkably [20]. Deng et al. obtained hierarchically interconnected porous $\mathrm{N}$-doped carbon through urea activation of cane sugar, which showed excellent specific capacitance $\left(350.8 \mathrm{~F} \mathrm{~g}^{-1}\right.$ at $1 \mathrm{~A}$ $\mathrm{g}^{-1}$ ) [21]. Although these materials show enhanced specific capacitance and cycle performance, their synthesis methods are usually complicated and expensive, making large-scale commercialization difficult. This necessitates selection of carbon material candidates to be combined with ZnNCN, which can be prepared using simple, quick and low-cost methods. Graphitized carbon nitride $\left(\mathrm{g}-\mathrm{C}_{3} \mathrm{~N}_{4}\right)$, a typical twodimensional carbon material, has been widely used in electrochemical process, due to its fast charge separation and relatively slow charge recombination characteristics during electrochemical process [22]. As compared to other $\mathrm{N}$-containing carbon materials, $\mathrm{g}-\mathrm{C}_{3} \mathrm{~N}_{4}$ has higher nitrogen content, which can provide more active reaction sites, increase electron donor/acceptor characteristics, improve mass transfer efficiency, and provide a large number of pseudocapacitance [23,24]. Notably, g- $\mathrm{C}_{3} \mathrm{~N}_{4}$ can be synthesized on a large scale from melamine or urea using fast and simple methods [25-27]. A low cost and customizable structure of $\mathrm{g}-\mathrm{C}_{3} \mathrm{~N}_{4}$ [28] make it ideal candidate for combining with $\mathrm{ZnNCN}$ to prepare a hybrid material with 2D morphology and high electron conductivity as a new generation supercapacitor electrode.

Herein, a metal-organic framework (MOF)-derived $\mathrm{ZnNCN} / \mathrm{g}-\mathrm{C}_{3} \mathrm{~N}_{4}$ composite synthesis, starting from truncated rhombic dodecahedral zeolitic imidazolate framework-8 (TRD-ZIF-8) precursor, is reported for the first time. Honeycomb-like porous $\mathrm{ZnNCN} / \mathrm{g}-\mathrm{C}_{3} \mathrm{~N}_{4}$ nanocomposites (HPZC) with high electrochemical activity were successfully prepared using a low-cost and easily upscalable synthesis method $[29,30]$. In order to alleviate previously reported collapsing of conventional zeolitic imidazolate framework during the carbonization process, resulting in a decrease in electrical conductivity and electrochemical performance $[31,32]$, a unique 3D-array stacked TRD-ZIF-8 material was synthesized in this study from zinc acetate and 2-methylimidazole, followed by the formation of a regular honeycomb-like porous structure of HPZC via addition of melamine and annealing at a high temperature under nitrogen atmosphere. The honeycomb-like porous structure prevents the degradation of electrochemical performance caused by the internal breakdown of the nanostructure, which is mainly attributed to directional etching of $\{110\}$ planes of TRD-ZIF- 8 by melamine. During this process, zinc ions were converted to $\mathrm{ZnNCN}$ nanoparticles with $\mathrm{g}-\mathrm{C}_{3} \mathrm{~N}_{4}$ evenly distributed on their surfaces. HPZC electrodes demonstrated excellent specific capacitance (5975 $\mathrm{F} \mathrm{g}^{-1}$ at $1 \mathrm{~A} \mathrm{~g}^{-1}$ ) and a high capacitance retention rate of $87.9 \%$ after 3000 deep charge-discharge cycles at $7 \mathrm{~A} \mathrm{~g}^{-1}$ with a decay rate of only $0.004 \%$. An asymmetric supercapacitor device (HPZC-4//AC ASC) was fabricated using this novel material, and demonstrated maximum power density and energy density of $7839 \mathrm{~W} \mathrm{Kg}^{-1}$ and $213 \mathrm{Wh} \mathrm{Kg}^{-1}$, respectively. Excellent electrochemical performance and long cycle life of HPZC can be attributed to $\mathrm{g}-\mathrm{C}_{3} \mathrm{~N}_{4}$ loaded on the surface of HPZC and unique honeycomb-like porous structure can efficiency improving ion diffusion efficiency and electrochemical charge storage kinetics. The study contributes to the development of low-cost preparation methods for TMNCN composites, possessing promising electrochemical properties, suitable for supercapacitor applications.

\section{Experimental section}

\subsection{Materials}

Analytical grade chemicals were used in this study without any further purification. $\mathrm{Zn}\left(\mathrm{CH}_{3} \mathrm{COO}\right)_{2} \cdot 2 \mathrm{H}_{2} \mathrm{O}$ (98\%), 2-methylimidazole (97\%), cetyltrimethylammonium bromide (CTAB) ( $\geq 98 \%$ ), zinc chloride $\left(\mathrm{ZnCl}_{2}, 98 \%\right)$, cyanamide $\left(\mathrm{H}_{2} \mathrm{NCN}, 95 \%\right)$, aqueous ammonia $\left(\mathrm{NH}_{4} \mathrm{OH}, 25 \mathrm{wt} \%\right)$, melamine (99\%), and ethanol ( $\left.\geq 99.7 \%\right)$ were purchased from Alfa Aesar.

\subsection{Synthesis of truncated rhombic dodecahedral zeolitic imidazolate framework-8 (TRD-ZIF-8) nanoparticles}

According to a typical synthesis procedure [33], 2-methylimidazole $(0.54 \mathrm{mM})$ and CTAB $(2.58 \mathrm{mM})$ were dissolved in $10 \mathrm{~mL}$ secondary distilled water to obtained solution A. $\mathrm{Zn}\left(\mathrm{CH}_{3} \mathrm{COO}\right)_{2} \cdot 2 \mathrm{H}_{2} \mathrm{O}(600 \mathrm{mg})$ was dissolved in $10 \mathrm{~mL}$ of water as solution $\mathrm{B}$. Subsequently, solution A and B were mixed well and gentle stirring for ca. 5-10 s to obtain white mixture. After standing for $3 \mathrm{~h}$ at room temperature, the white solid was concentrated from the mixture at $9,000 \mathrm{rpm}$ in $50 \mathrm{~mL}$ Falcon tubes and then washed three-four times with deionized water. Collected wet pellets were dried in a vacuum drying box overnight at $60^{\circ} \mathrm{C}$ to obtain TRDZIF-8 nanoparticles in the form of white powder.

\subsection{Synthesis of honeycomb-like porous $\mathrm{ZnNCN} / \mathrm{g}-\mathrm{C}_{3} \mathrm{~N}_{4}$ (HPZC) nanocomposites}

The obtained TRD-ZIF-8 nanoparticles ( $2 \mathrm{~g})$ and melamine $(2 \mathrm{~g})$ were mixed during 5-10 min using a vortex oscillator and transferred into a $100 \mathrm{~mL}$ alumina crucible equipped with a lid. The crucible was put into a muffle furnace filled with nitrogen which was heated to $550^{\circ} \mathrm{C}$ at a rate of $3^{\circ} \mathrm{C} \mathrm{min}^{-1}$ and kept at this temperature for $3 \mathrm{~h}$. After the furnace naturally cooled down to a room temperature, HPZC was collected in the form of black powder. For comparison, TRD-ZIF-8/melamine combinations with mass ratios 5:1, 1:1, 1:5, 1:8 and 1:10 were mixed to produce HPZC-1, HPZC-2, HPZC-3, HPZC-4 and HPZC-5 reference materials, respectively.

\subsection{Synthesis of $Z n N C N / C$ (ZC) nanocomposites}

ZnNCN/C (ZC) material not containing $g-\mathrm{C}_{3} \mathrm{~N}_{4}$ phase was synthesized for comparison, using the same preparation method as for HPZC, except for the addition of melamine before the calcination process.

\subsection{Synthesis of ZnNCN powder}

ZnNCN reference material was synthesized according to previously reported procedure [12]. Zinc chloride (3.9 g), cyanamide (2.05 g), 25 $\mathrm{wt} \%$ aqueous ammonia $(12 \mathrm{~mL})$ and distilled water $(50 \mathrm{~mL})$ were mixed and stirred for $3 \mathrm{~h}$. The obtained white precipitate was filtered and washed with distilled water several times. Finally, the white powder was obtained after drying in a vacuum at $80^{\circ} \mathrm{C}$ overnight.

\subsection{Physical characterization and electrochemical measurements}

The measurements of field-emission scanning electron microscopy (FE-SEM), transmission electron microscopy (TEM), fourier-transform infrared spectroscopy (FT-IR), X-ray Photoelectron Spectroscopy (XPS), Brunauer-Emmett-Teller (BET), Raman spectra (Thermo Nicolet Almega XR) and powder X-ray diffraction (PXRD) have been used in this paper. Details are shown in supporting information.

CHI 660E electrochemical workstation and LAND CT2001A battery measurement system have been used for electrochemical measurements. Briefly, a three-electrodes system has been used with HPZC as a working electrode (WE), active carbon (AC) as a counter electrode (CE) and $\mathrm{Hg}$ / 
TRD-ZIF-8

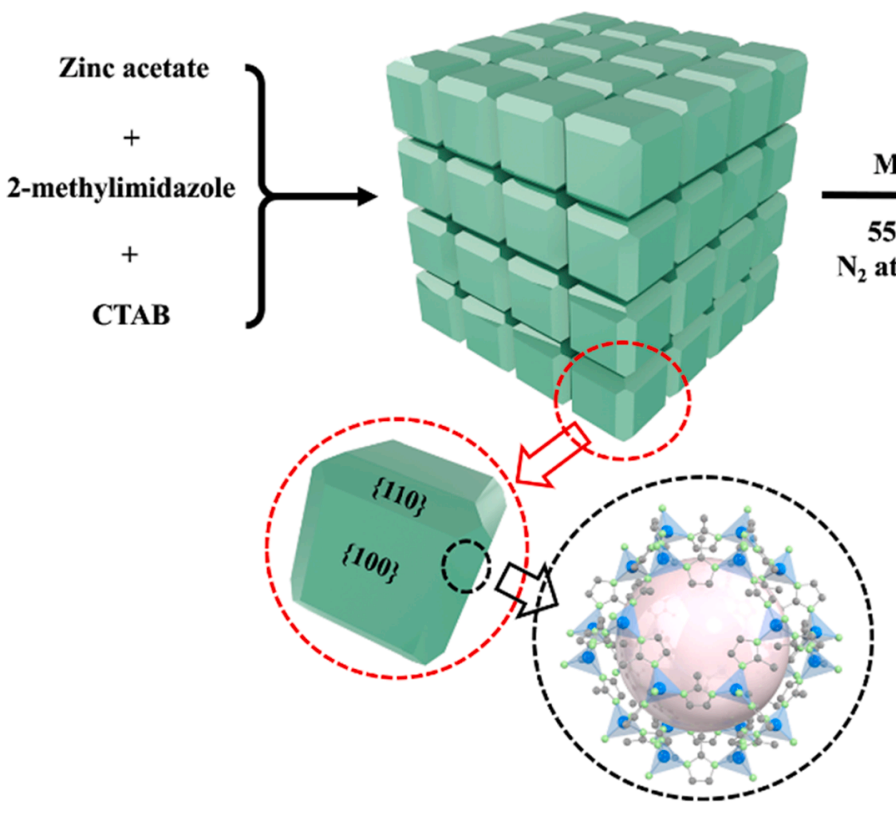

HPZC

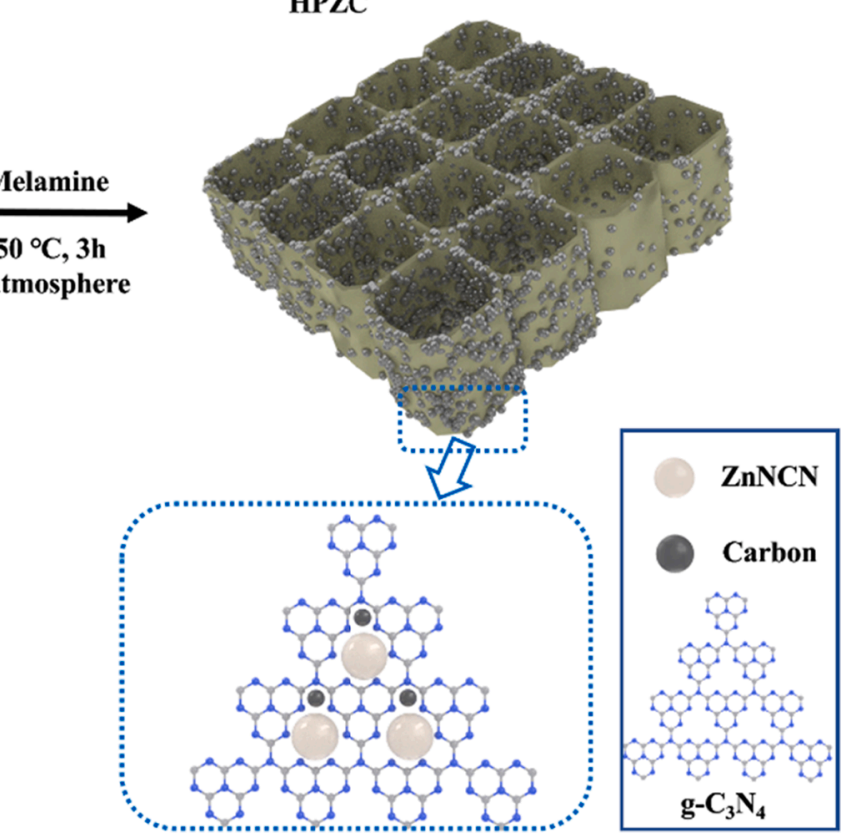

Scheme 1. Schematic diagram of formation of honeycomb-like porous $\mathrm{ZnNCN} / \mathrm{g}-\mathrm{C}_{3} \mathrm{~N}_{4}$ (HPZC) nanocomposites.

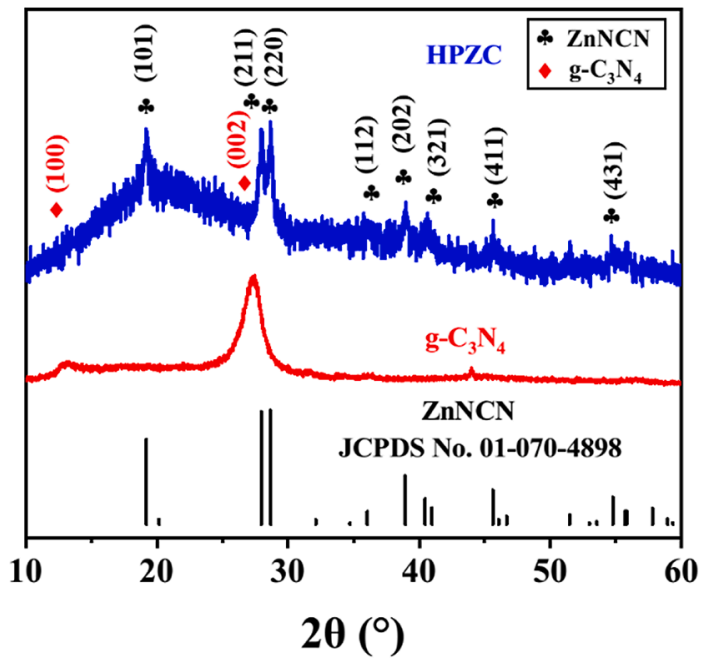

Fig. 1. The XRD patterns of simulated $\mathrm{ZnNCN}$ powder, pure g- $\mathrm{C}_{3} \mathrm{~N}_{4}$ powder and HPZC nanocomposites.

$\mathrm{HgO}$ as a reference electrode (RE), respectively. Detailed electrode manufacturing and testing and calculation methods also seen in supporting information.

\section{Results and discussion}

Honeycomb-like porous nanocomposites $\mathrm{ZnNCN} / \mathrm{g}-\mathrm{C}_{3} \mathrm{~N}_{4}$ (HPZC) were prepared using a two-step process. Firstly, a previously reported method [33] involving the use of dual capping ligands of 2-methylimidazole and CTAB, was followed to synthesize monodisperse TRDshaped ZIF-8 parent colloids. As shown in Scheme 1, the TRD-ZIF-8 parent colloids contain 6 square $\{100\}$ planes and 12 hexagonal $\{110\}$ planes, making it a rhombic dodecahedron with six truncated corners. Second, melamine was added as both a sacrificial template and a catalyst to yield array-shaped honeycomb porous nanocomposite (HPZC), with $\mathrm{g}-\mathrm{C}_{3} \mathrm{~N}_{4}$ nanosheets and ZnNCN nanoparticles formed on its surface, using high temperature annealing under nitrogen atmosphere.
Low cost precursors and simple synthetic processes were used in this study in order to allow for future scaling up of the material.

The X-ray diffraction pattern of ZnNCN, g- $\mathrm{C}_{3} \mathrm{~N}_{4}$ and HPZC are presented in Fig. 1. Two diffraction peaks at ca. $27.4^{\circ}$ and $13.1^{\circ}$ are characteristic of $\mathrm{g}-\mathrm{C}_{3} \mathrm{~N}_{4}$ where the peak at ca. $27.4^{\circ}$ corresponds to $\{002\}$ crystal plane, which is related to the interlayer stacking structure of aromatic systems [34], and the peak at ca. $13.1^{\circ}$ represents interplanar repeating units of g- $\mathrm{C}_{3} \mathrm{~N}_{4}$ [35]. Diffraction peaks at ca. $19.2^{\circ}, 27.9^{\circ}$, $28.6^{\circ}, 36.0^{\circ}, 38.9^{\circ}, 40.5^{\circ}, 45.6^{\circ}$ and $54.7^{\circ}$ correspond to $\{101\},\{211\}$, $\{220\},\{112\},\{202\},\{112\},\{202\},\{321\},\{411\}$ and $\{431\}$ planes of ZnNCN, respectively, with lattice parameters $\mathrm{a}=\mathrm{b}=8.8047 \AA$ and $\mathrm{c}$ $=5.4329 \AA$ (JCPDS No. 01-070-4898) [12]. A wide peak between ca. $15^{\circ}$ and $25^{\circ}$ is attributable to amorphous carbon, which significant presence weakens the signals in $\mathrm{g}-\mathrm{C}_{3} \mathrm{~N}_{4}$ and $\mathrm{ZnNCN}$, and is additionally confirmed by black color of HPZC as shown in Fig. S1.

Fig. 2a shows the FT-IR spectra of pure $\mathrm{g}-\mathrm{C}_{3} \mathrm{~N}_{4}$, and various HPZC nanocomposites with different g- $\mathrm{C}_{3} \mathrm{~N}_{4}$ loading. For $\mathrm{g}-\mathrm{C}_{3} \mathrm{~N}_{4}$, the peak at ca. $1651 \mathrm{~cm}^{-1}$ can be ascribed to the $\mathrm{C}=\mathrm{N}$ stretching and the peaks at ca. 1396, 1558 and $1651 \mathrm{~cm}^{-1}$ can be assigned to the heptazine-derived repeating units [36]. Moreover, the peaks at ca. $1241 \mathrm{~cm}^{-1}$ and ca. 807 $\mathrm{cm}^{-1}$ may be assigned to the $\mathrm{C}-\mathrm{N}$ stretching mode and the out of plane breathing vibration characteristic of s-triazine ring system of $\mathrm{g}-\mathrm{C}_{3} \mathrm{~N}_{4}$, respectively [37]. For HPZC, the newly emerged IR peak at $2050 \mathrm{~cm}^{-1}$ are attributed to asymmetrical stretching vibration of characteristic - $\mathrm{N}$ $=\mathrm{C}=\mathrm{N}$ - bonding in ZnNCN, and the peak at ca. $567 \mathrm{~cm}^{-1}$ can be assigned to its doubly degenerated deformation mode [38]. Moreover, all the HPZC samples have presented the main characteristic peaks of $g$ $\mathrm{C}_{3} \mathrm{~N}_{4}$ and these peaks (ca. $1645 \mathrm{~cm}^{-1}$ and $1238 \mathrm{~cm}^{-1}$ ) show slight red shift compared with pure $\mathrm{g}-\mathrm{C}_{3} \mathrm{~N}_{4}$, indicating the existence of intense interaction between $\mathrm{g}-\mathrm{C}_{3} \mathrm{~N}_{4}$ and $\mathrm{ZnNCN}$ rather than a simply physical adsorption [39], and this chemical bond also suggests that of great significance for the charge transfer and stability of this specific structure.

Raman spectrum has been used to further study the internal chemical environment of HPZN. The peak at ca. $1371 \mathrm{~cm}^{-1}$ can be related to the asymmetric $-\mathrm{N}=\mathrm{C}=\mathrm{N}$ - stretching mode (Fig. 2b) [40]. The peaks at ca. $758 \mathrm{~cm}^{-1}$ and $1265 \mathrm{~cm}^{-1}$ may be assigned to the bending mode of $-\mathrm{N}=$ $\mathrm{C}=\mathrm{N}$ - group. The signal of the $-\mathrm{N}=\mathrm{C}=\mathrm{N}$ - bending $\left(\delta_{\text {Raman }}=758 \mathrm{~cm}^{-1}\right)$ occurs at higher wavenumbers compared to FT-IR $\left(\delta_{\mathrm{IR}}=567 \mathrm{~cm}^{-1}\right)$, which may due to the mixing of vibrational states (Fermi resonance) 
(a)

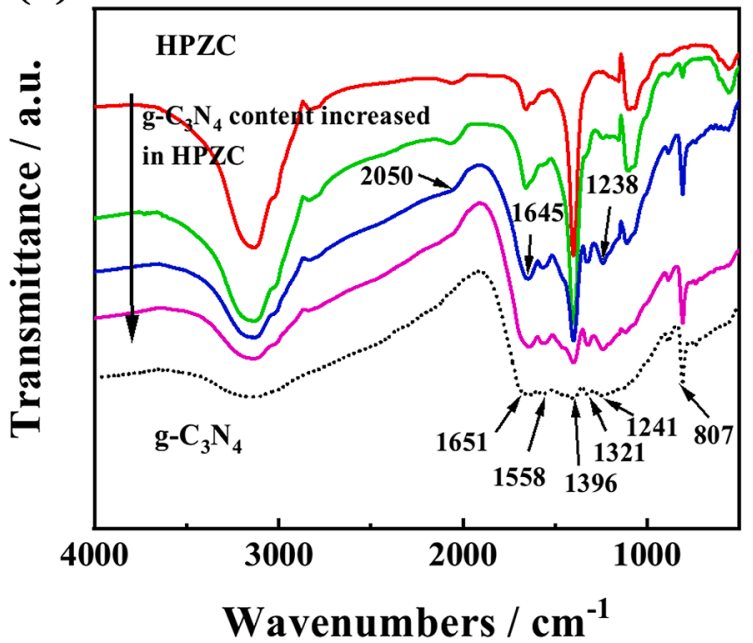

(b)

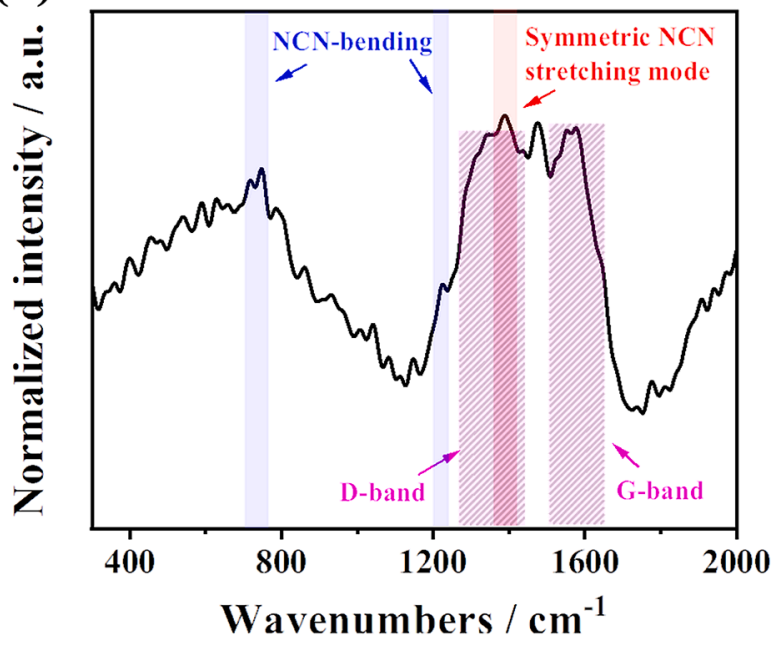

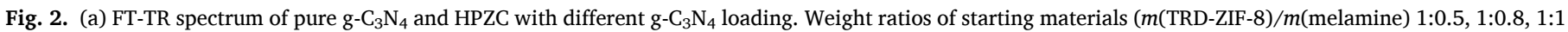

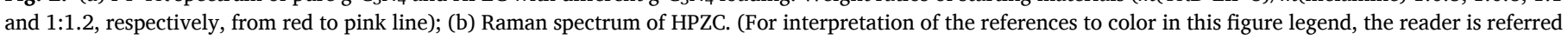
to the web version of this article.)

[41]. Moreover, the characteristic peaks of the ZnNCN are located below $500 \mathrm{~cm}^{-1}$, which can be attributed to the lattice vibration. Besides, two broad graphitic peaks at ca. $1354 \mathrm{~cm}^{-1}$ and $1589 \mathrm{~cm}^{-1}$ have been observed, which are attributed to the D-band and G-band of $\mathrm{g}-\mathrm{C}_{3} \mathrm{~N}_{4}$, respectively. Specifically, D-band can be assigned to the disordered structure on graphite plane, and G-band can be assigned to the $\mathrm{E}_{2 \mathrm{~g}} \mathrm{vi}$ bration mode in $\mathrm{sp}^{2}$ graphitic carbon [42]. These results further illustrate the presence of $\mathrm{g}-\mathrm{C}_{3} \mathrm{~N}_{4}$ and $\mathrm{ZnNCN}$ in $\mathrm{HPZC}$ nanocomposites.

The morphology and microstructure structure of TRD-ZIF-8 and HPZC were characterized by scanning electron microscopy (SEM) and transmission electron microscopy (TEM). As shown in Fig. 3a and b, the as-prepared TRD-ZIF-8 nanoparticles show truncated rhombic dodecahedral (TRD) nanostructures, and the average particle size is around $210 \pm 20 \mathrm{~nm}$. Due to TRD-ZIF-8 colloidal precursor, the resulting TRDZIF-8 particles revealed the self-assembled superstructures along the three directions of space [29]. After adding melamine and calcining at a high temperature under nitrogen atmosphere, the original solid arraylike nanostructure is destroyed and changes to a honeycomb-like porous structure with the rough wall surface (Fig. $3 \mathrm{c}$ and d). The grayscale contrast in TEM images (Fig. $3 e$ and f) indicates that the obtained HPZC nanocomposites are composed of carbon, $\mathrm{ZnNCN}$ and $\mathrm{g}-\mathrm{C}_{3} \mathrm{~N}_{4}$. The lattice fringe with spacing of $1.95 \mathrm{~nm}$, corresponding to \{211\} crystal plane of ZnNCN, is recorded in the HRTEM images by snapshot mode, which consistent with the XRD results. Moreover, g- $\mathrm{C}_{3} \mathrm{~N}_{4}$ layer is uniforms distributed on the surface of HPZC, which may be contribute to the thermal decomposition of melamine (Fig. 3f). Besides, it is clearly that the interfaces between $\mathrm{ZnNCN} /$ carbon and $\mathrm{g}-\mathrm{C}_{3} \mathrm{~N}_{4}$ is seamlessly contacted, which may due to the in-situ forming process of $\mathrm{g}-\mathrm{C}_{3} \mathrm{~N}_{4}$ [43]. Energy-dispersive X-ray spectroscopy (EDS) and elemental mapping were performed in Fig. 2h-l. The uniform distribution of $\mathrm{C}, \mathrm{N}$ and $\mathrm{Zn}$ further suggests the homogenous distribution of $\mathrm{ZnNCN}$ and $\mathrm{g}_{-} \mathrm{C}_{3} \mathrm{~N}_{4}$ in HPZC.

In order to study the formation mechanism of this honeycomb-like porous structure, the ZC samples without adding melamine were synthesized for comparison. As shown in Fig. S2, the TRD structure is roughly kept in the obtained $\mathrm{ZC}$, and the smooth surface becomes rough. Moreover, massive nanoparticles appeared on the surface, which may be contributed to small organic molecular in TRD-ZIF-8 changed into carbon particles and zinc ion changed into ZnNCN nanoparticles after calcining process [44]. Compared the morphology of HPZC with that of $\mathrm{ZC}$, in our opinion, melamine may play the key role in the formation of honeycomb-like porous structure, which may due to that melamine set as a self-sacrifice agent apart from as the etchant $[45,46]$. Specifically, as shown in Scheme S1, TRD-ZIF-8 particles show regular $\{110\}$ and $\{100\}$ planes. However, due to the action of surfactant (CTAB), TRDZIF-8 nanoparticles can self-assembly into the array structure, resulting in $\left\{\begin{array}{lll}1 & 0 & 0\end{array}\right\}$ planes on adjacent TRD-ZIF-8 nanoparticles overlap with each other. Thus, the added melamine only covers on non-overlapping $\{100\}$ planes instead of that of overlapped ones. During the calcined process, melamine, as the etchant, corrodes these non-overlapping $\{100\}$ planes, resulting in the formation of honeycomb-like porous backbones [47]. Moreover, the $\mathrm{g}-\mathrm{C}_{3} \mathrm{~N}_{4}$ is produced at the same time and adhered to the porous wall and formed the final structure. This unique formation mechanism may provide new ideas for the preparation of large-scale porous structures.

The $\mathrm{N}_{2}$ sorption analysis is carried out to investigate the porous nature of HPZC. As shown in Fig. S3, the $\mathrm{N}_{2}$ sorption isotherm corresponding to HPZC is typically of type IV (BDDT classification), showing its mesoporous characteristic. Moreover, the $\mathrm{H} 3$ type shape at higher relative pressure of hysteresis loop suggests the existence of similar slitlike pores, which may be originated from $\mathrm{g}-\mathrm{C}_{3} \mathrm{~N}_{4}$ layers stacking [48]. Barrett-Joyner-Halenda (BJH) pore-size distribution analyses confirms that diameters of mesopores cover the wide range from 2 to $30 \mathrm{~nm}$. As shown in Table S1, BET surface area of HPZC $\left(80.8 \mathrm{~m}^{2} \mathrm{~g}^{-1}\right)$ much higher than that of bulk $\mathrm{g}-\mathrm{C}_{3} \mathrm{~N}_{4}\left(9 \mathrm{~m}^{2} \mathrm{~g}^{-1}\right)$ [49] and many related g- $\mathrm{C}_{3} \mathrm{~N}_{4}$ based materials $[50,51]$, which can contribute to the specific honeycomb-like porous structure in HPZC.

According to the results of the thermogravimetric analysis (TGA) under nitrogen atmosphere (Fig. S4), it is obviously that there is a slow weight decline at low temperature (less than $600{ }^{\circ} \mathrm{C}$ ), which may be contribute to the existence of $\mathrm{g}-\mathrm{C}_{3} \mathrm{~N}_{4}$ steam in the mesopores of HPZC. Moreover, a rapid decline appeared between $600{ }^{\circ} \mathrm{C}$ and $800{ }^{\circ} \mathrm{C}$, which may due to the rapid loss of $\mathrm{g}-\mathrm{C}_{3} \mathrm{~N}_{4}$ at high temperature [52]. All these results are consistent with above XRD and BET analysis.

The X-ray photoelectron spectroscopy (XPS) is used to further analyze the chemical composition and the surface chemical states of HPZC. The XPS survey spectra are shown in Fig. 4a and the main peaks can be assigned to $\mathrm{Zn}, \mathrm{C}$ and $\mathrm{N}$, respectively. As shown in Fig. 4b, C1s spectra can be fitted into two peaks at ca. $284.7 \mathrm{eV}$ and $286.5 \mathrm{eV}$, which can be ascribed to the $\mathrm{C}-\mathrm{C}$ bond of adventitious carbon and $\mathrm{C}-\mathrm{N}$ bonding in the tri-s-triazing ring or the -N-C-N- carbodiimides, respectively [53]. Moreover, no peak appeared at ca. $282 \mathrm{eV}$, indicating that there is no direct bond between $\mathrm{C}$ and $\mathrm{Zn}(\mathrm{C}-\mathrm{Zn})$ in the nanocomposites [54], all zinc atoms should be in ZnNCN nanoparticles. For N1s spectra, the 

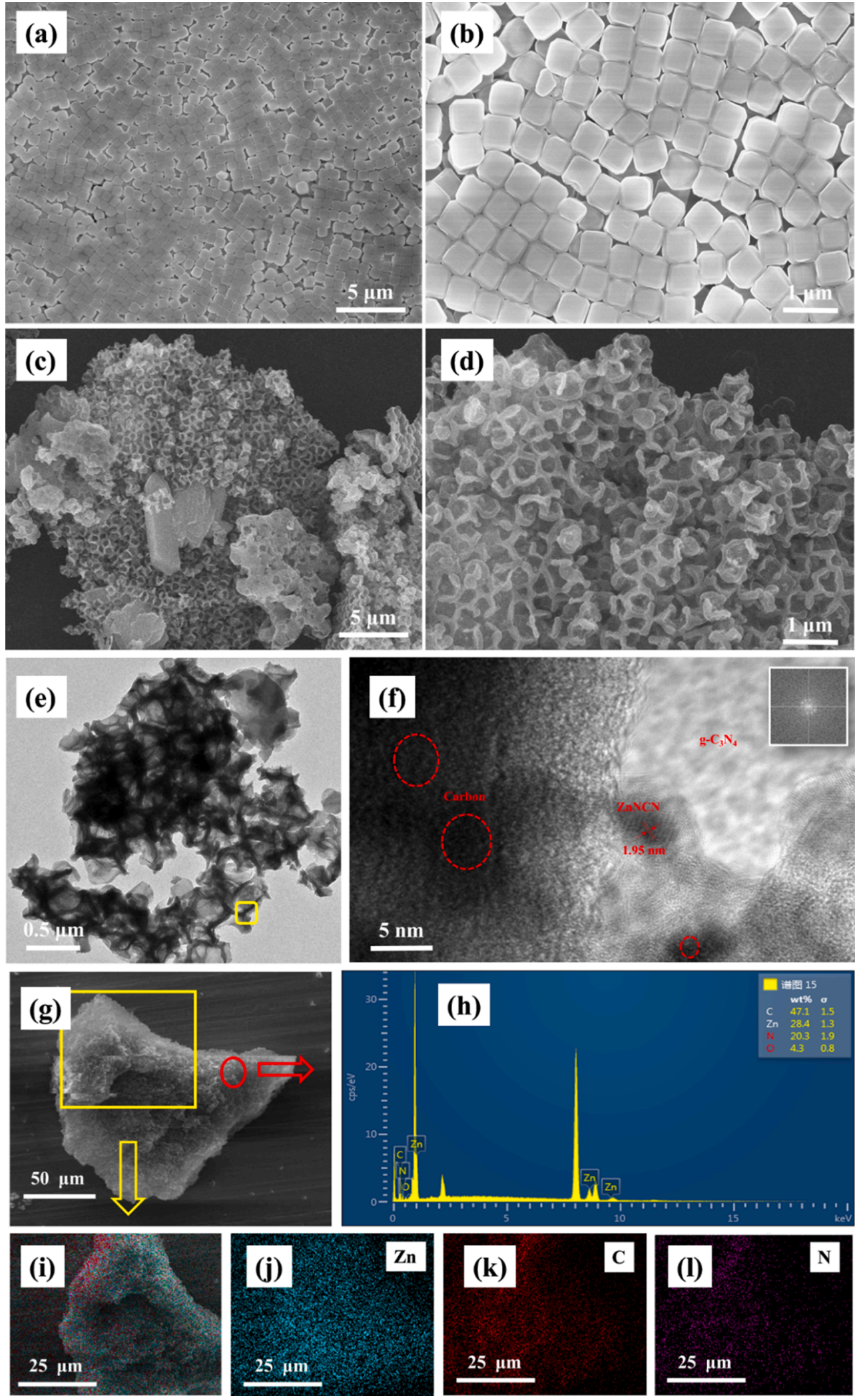

(h)
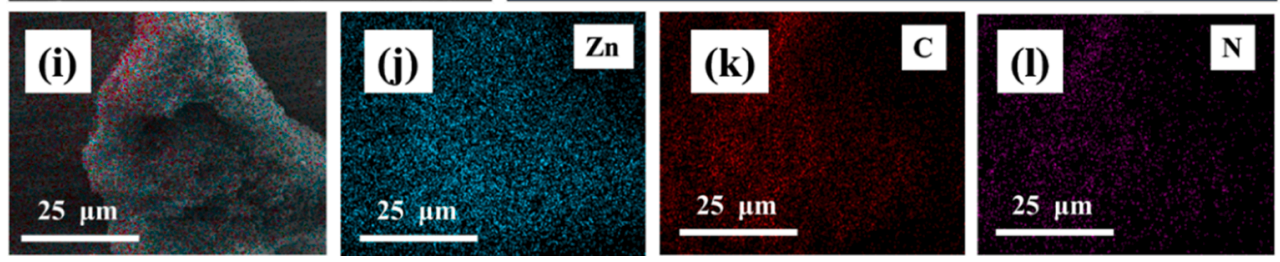

Fig. 3. SEM and HRSEM images of (a) (b) array-like TRD-ZIF-8 particles and (c) (d) HPZC nanocomposites at different scale bars; (e) TEM image of HPZC and (f) corresponding HRTEM image in yellow box area, insert showing the crystal diffraction for $\mathrm{ZnNCN}$; (g) SEM image of HPZC and (h) the corresponding EDAX pattern in red cycle area; (i) the enlarged SEM image of Fig. ( $g$ ) in yellow box region, corresponding elemental mapping images of (j) Zn, (k) C and (l) N, respectively. (For interpretation of the references to color in this figure legend, the reader is referred to the web version of this article.)

region can be divided into two energies, which can be ascribed to $\mathrm{N}-(\mathrm{C})_{3}$ (ca. $398.4 \mathrm{eV}$ ) and C-N-(H) 2 groups (ca. $400.4 \mathrm{eV}$ ), respectively [55]. For $\mathrm{Zn} 2 p$ spectra for HPZC, two characteristic peaks can be assigned to $\mathrm{Zn} 2 p 3 / 2$ (ca. $1022.0 \mathrm{eV}$ ) and Zn2p1/2 (ca. $1045.1 \mathrm{eV}$ ), respectively [38]. However, the binding energy of Zn2p of HPZC are slightly lower than that of TRD-ZIF-8 (Fig. S5), which may due to the unique Z-scheme heterostructure formed at the $\mathrm{g}-\mathrm{C}_{3} \mathrm{~N}_{4} / \mathrm{ZnNCN}$ interface[13], indicating the enhanced interfacial charge transfer and redistribution abilities.

The electrochemical performance of commercial $\mathrm{ZnNCN}, \mathrm{ZC}$ and HPZC nanocomposites as working electrodes of supercapacitors was 
(a)

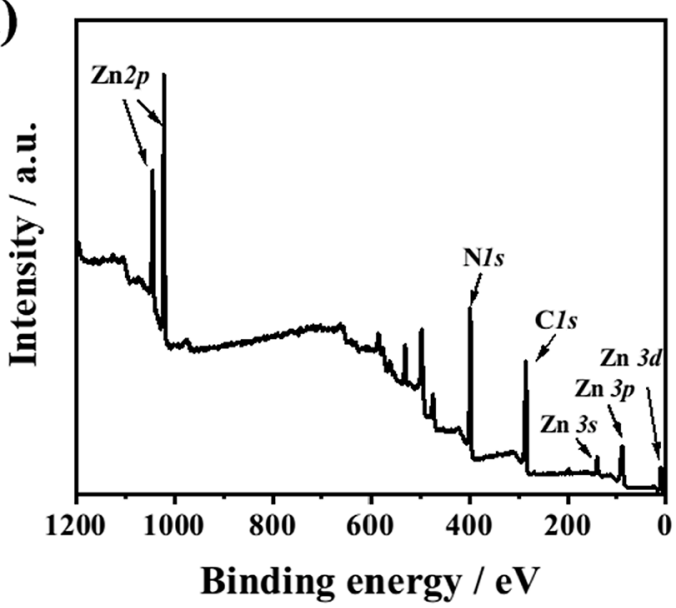

(c)

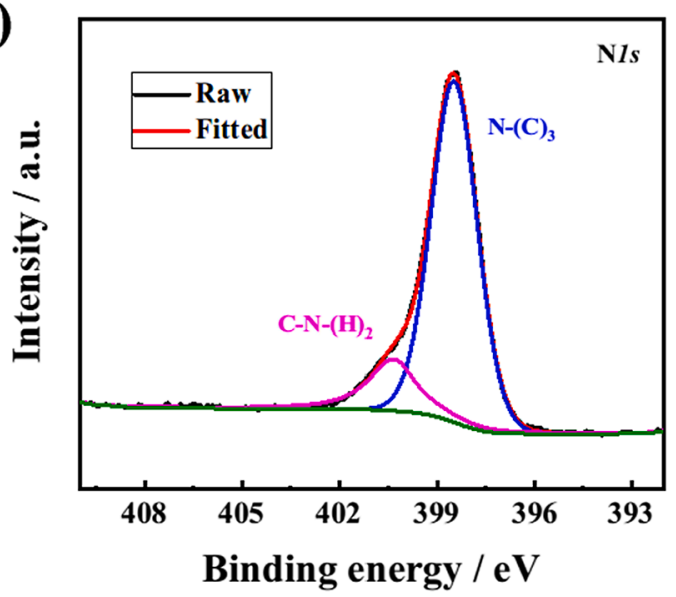

(b)

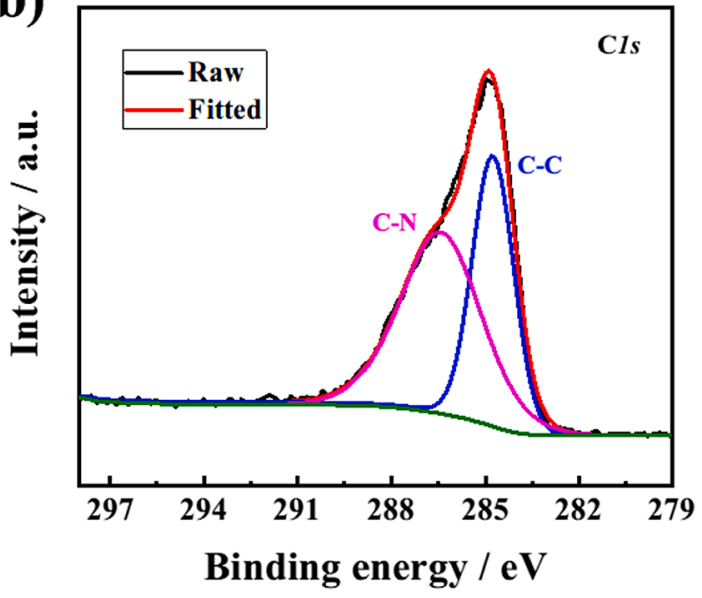

(d)

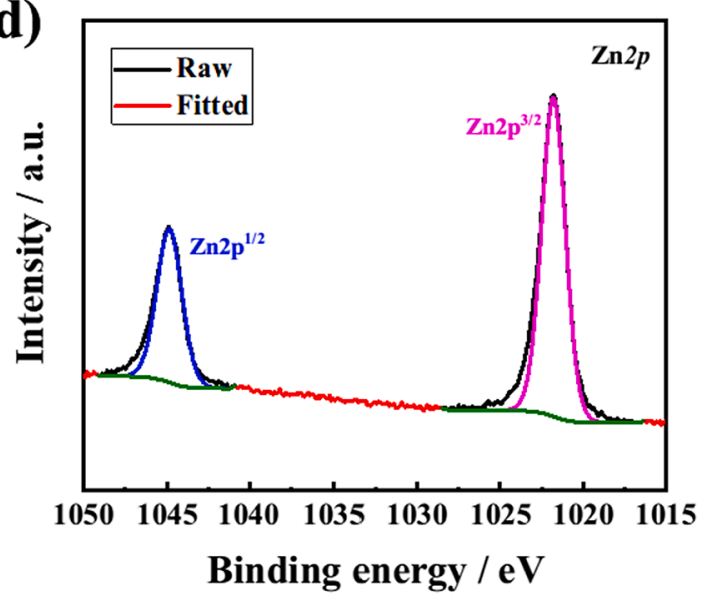

Fig. 4. (a) XPS survey spectrum of HPZC and corresponding high-resolution (b) C1s, (c) N1s and (d) Zn2p XPS spectra.

tested in a three-electrode system with $3 \mathrm{M} \mathrm{KOH}$ as the electrolyte. Since all samples in this paper are attached and measured on nickel foam substrates, the effects of nickel foam should be firstly considered. Noticeably, many literatures $[56,57]$ report that the slight current was recorded on the bare nickel foam electrode, thus it would be reasonable to ignore the capacitance contribution of nickel foam. The cyclic voltammetry (CV) curves of commercial ZnNCN and HPZC are shown in Fig. 5a. The redox peaks in ZnNCN and HPZC suggest the nature of typical pseudocapacitive behavior. Moreover, the redox peak positions of ZnNCN at ca. $0.3 \mathrm{~V}$ and $0.45 \mathrm{~V}$, respectively, are approximately consistent with that of HPZC. However, the cathode potential (ca. 0.35 $\mathrm{V}$ ) of ZnNCN is slightly slower than that of HPZC (ca. $0.36 \mathrm{~V}$ ), which may be attributed to the energy levels of $\mathrm{ZnNCN} / \mathrm{g}-\mathrm{C}_{3} \mathrm{~N}_{4}$ heterojunction in HPZC different with that of ZnNCN [58].

HPZC electrodes show the same symmetrical redox peaks at different scan rates (range from $1 \mathrm{mV} \mathrm{s}^{-1}$ to $30 \mathrm{mV} \mathrm{s}^{-1}$ ), indicating that the HPZC have excellent rate capability and relatively low resistance (Fig. 5b). It is well known that both a surface and a bulk play important role in redox activity under a low discharge current density, while under a high discharge current density it is surface activity that becomes dominant. Thus, a linear relationship between square root of a scan rate and peak current density further proves the occurrence of a Faradaic redox reaction on the electrode surface (Fig. S6). Moreover, CVs can also provide greater insight into the charge storage kinetics of HPZC electrode. Generally, the following equation proposed by Lindström et al can be used to depicted the relationship between the san rates $(v)$ and peak current $(i):[59,60]$
$i(V)=a v^{b}$

where $i(V)$ is the current at a specific potential, a and $\mathrm{b}$ are adjustable parameters. Specifically, kinetic limitations can be estimated from the limiting cases of the $b$-value; $b=0.5$, which suggests a diffusioncontrolled (or battery-like) behavior, caused by slowly semi-infinite linear ion diffusion; $\mathrm{b}=1.0$, which indicates a capacitive behavior related to a fast faradaic redox reaction on the surface. [59,60] According to the calculation of CVs curves with the scan rate ranges from 1 to $30 \mathrm{mV} \mathrm{s}^{-1}$ (Fig. 5b), the b-values for anodic and cathodic peaks of HPZC electrode are 0.72 and 0.70 , respectively (Fig. S7). These b-values are between 0.5 and 1 , which suggests that this electrochemical reaction is both surface-controlled and diffusion-controlled. However, till now, no research on the electron transport mechanism of ZnNCN system was reported, thus, the internal electrochemical mechanism still needs follow-up research.

Electrochemical performance of HPZC materials was further studied utilizing a series of HPZC-n electrodes where $n$ signifies the ratio of TRDZIF-8 to melamine used in the synthesis as described in the Experimental section. Fig. $5 c$ presents the galvanostatic charge-discharge (GCD) curves of HPZC-n series electrodes at $1 \mathrm{~A} \mathrm{~g}^{-1}$. These non-linear charge curves and their corresponding discharge curves are nearly symmetrical, which combined the CV results to prove the pseudocapacitive behavior of HPZC electrodes during these electrochemical processes [3]. Among these materials, HPZC-4 has the longest discharge time. After calculation based on the peak areas and IR-drop correction, the discharge specific capacitance of HPZC-4 is ca. $5975 \mathrm{~F} \mathrm{~g}^{-1}$ at $1 \mathrm{~A} \mathrm{~g}^{-1}$, which is distinctly superior than other HPZC samples (i.e., HPZC-1 (ca. $2405 \mathrm{~F} \mathrm{~g}^{-1}$ ), HPZC- 
(a)

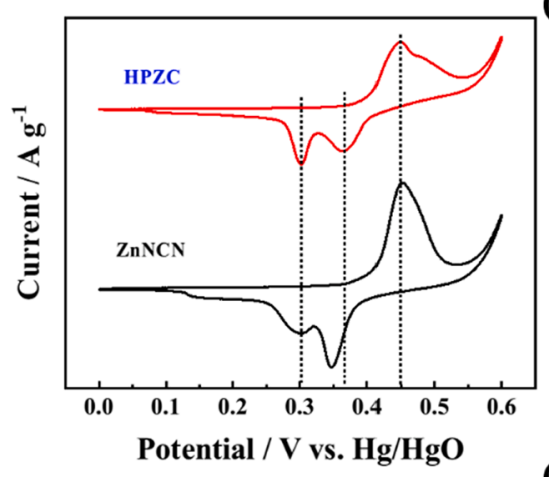

(c)

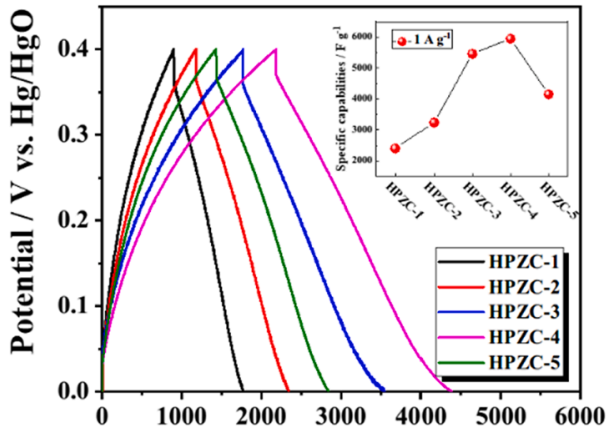

(e)

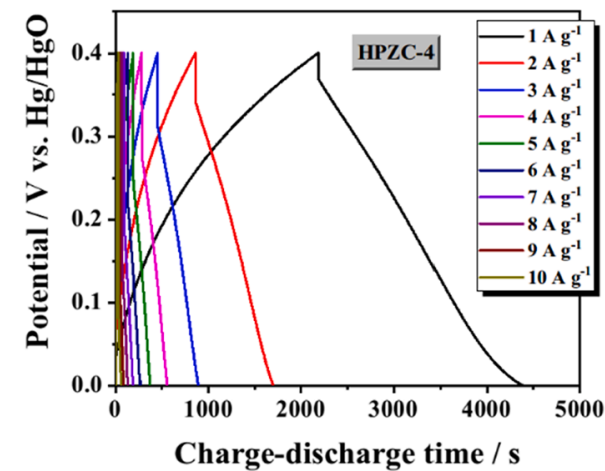

(g)

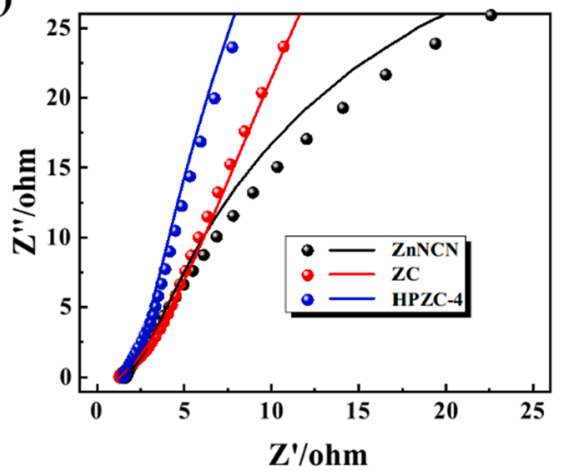

(i)

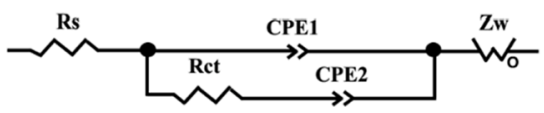

(b)

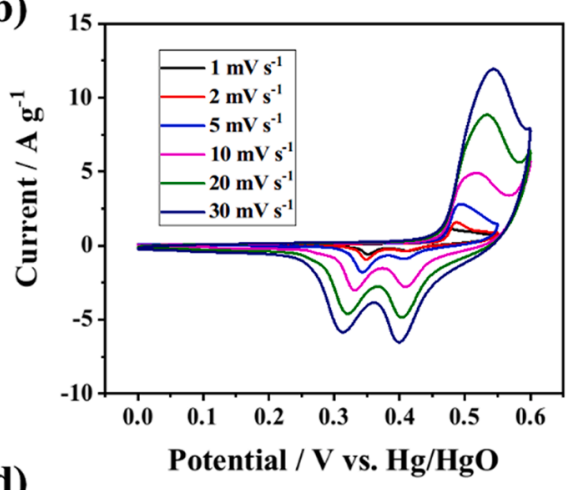

(d)

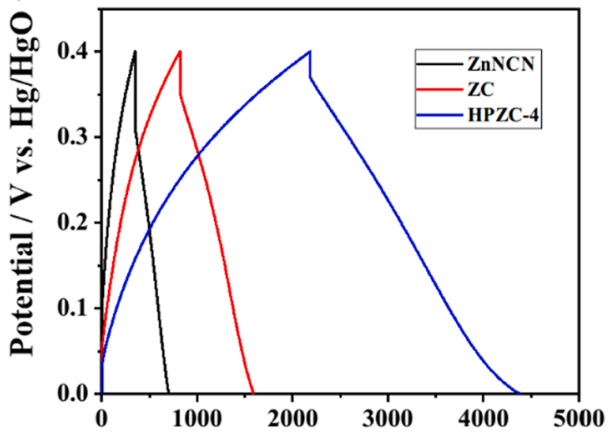

(f)

Charge-discharge time / s

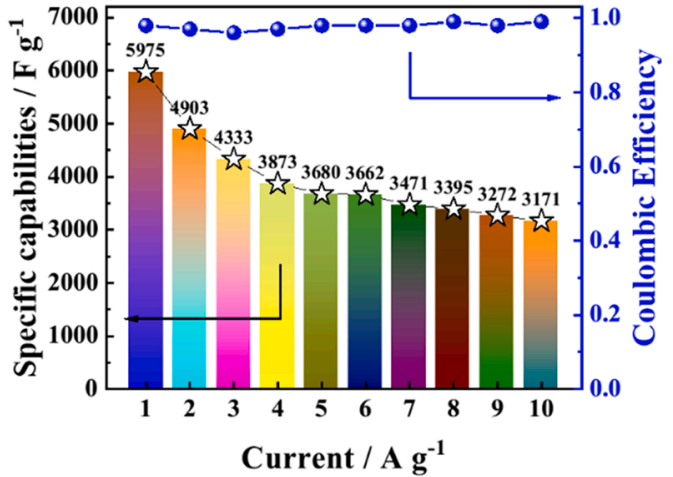

(h)

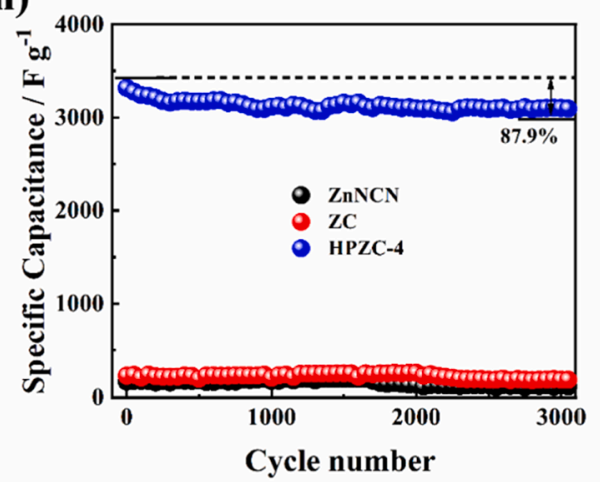

Fig. 5. (a) CV curves of pure $\mathrm{ZnNCN}$ powder and HPZC with the scan rate at $5 \mathrm{mV} \mathrm{s}^{-1}$; (b) CV curves of HPZC at various scan rates,; (c) GCD curves of HPZC-n with various mass ratios of starting materials ( $m$ (TRD-ZIF-8) $/ m$ (melamine)) at $1 \mathrm{~A} \mathrm{~g}^{-1}$, and insert figure shows the specific capacitance of HPZC series electrodes at $1 \mathrm{~A} \mathrm{~g}^{-1}$; (d) GCD curves of ZnNCN, ZC and HPZC-4 at $1 \mathrm{~A} \mathrm{~g}^{-1}$; (e) GCD curves of HPZC-4 scanned at various current densities, (f) corresponding specific capacitance of HPZC-4 after IR- drop correction and Coulombic efficiency of HPZC-4; (g) Nyquist plots (dot) and corresponding fitting line of ZnNCN, ZC and HPZC-4, (i) equivalent circuit used to fit impedance spectra; (h) cycling stability of ZnNCN, ZC and HPZC-4 at the current density of $7 \mathrm{~A} \mathrm{~g}^{-1}$. 
(a)

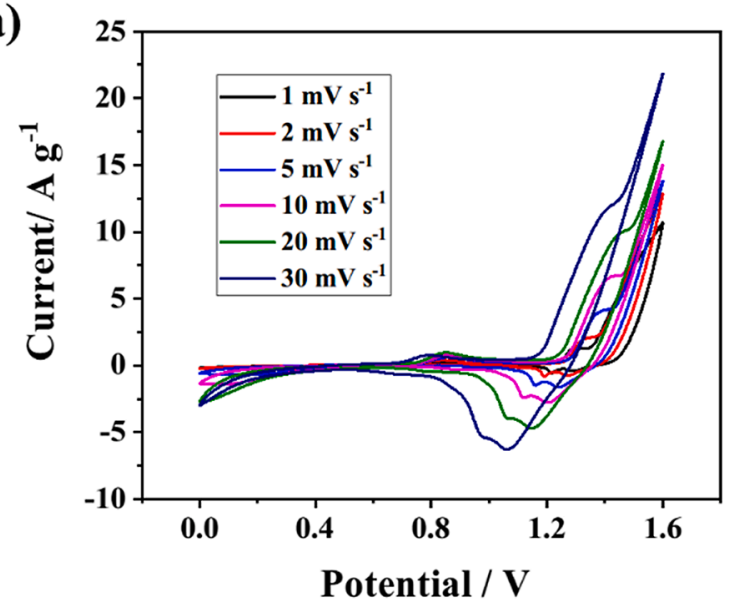

(b)

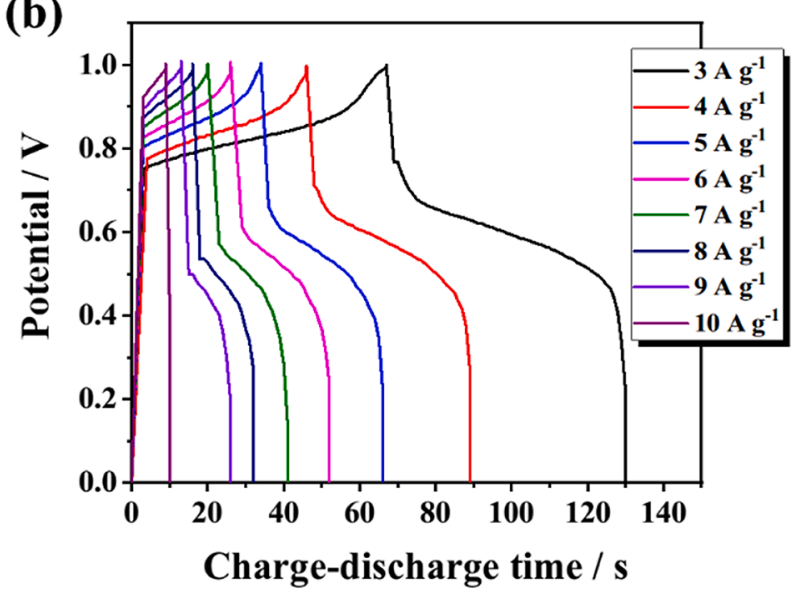

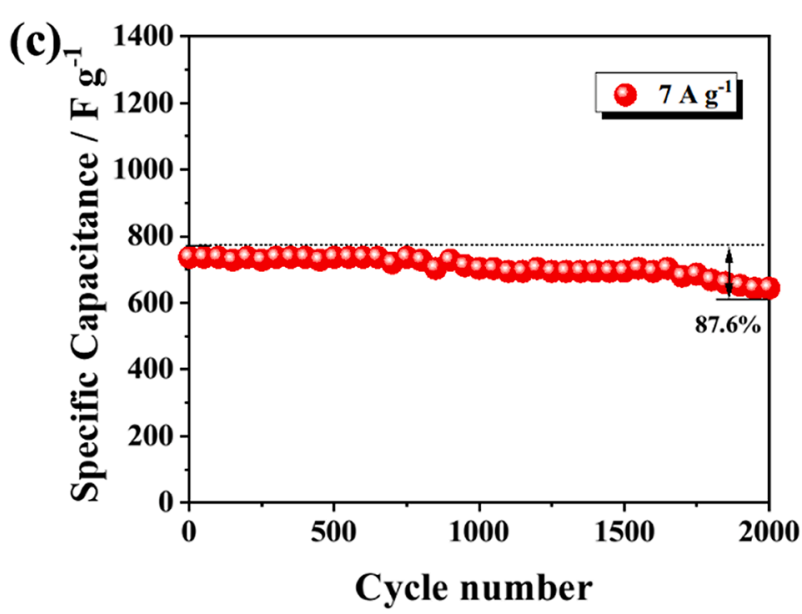

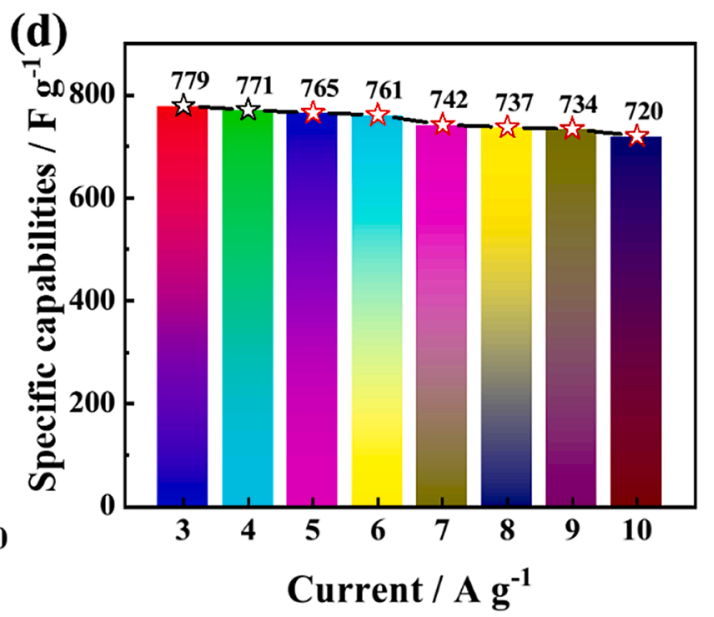

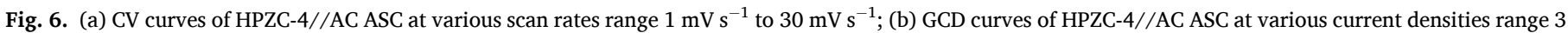
$\mathrm{A} \mathrm{g}^{-1}$ to $10 \mathrm{~A} \mathrm{~g}^{-1}$, (d) corresponding specific capacitance after IR drop correction; (c) cycling performance of HPZC-4//AC ASC at 7 A g ${ }^{-1}$;

that of $\mathrm{ZnNCN}$ at $1 \mathrm{~A} \mathrm{~g}^{-1}$, which can be attributed to the high conductivity of carbon materials in ZC [2]. Moreover, the discharge time of HPZC- 4 shows 3 time than that of ZC, respectively, indicating that the existence of $\mathrm{g}-\mathrm{C}_{3} \mathrm{~N}_{4}$ on the surface of HPZC would be contribute to the improvement of capacitance [28].

High rate capability is another important parameter for supercapacitors. HPZC-4 electrodes are measured with the current density range from $1 \mathrm{~A} \mathrm{~g}^{-1}$ to $10 \mathrm{~A} \mathrm{~g}^{-1}$ as shown in Fig. 5e. The results show that charge and discharge curves are nearly similar at various current densities, suggesting the highly reversible redox reaction occurs on the electrode surface with high Coulombic efficiency $(\geq 95 \%)$, which consistent with the results shown in Fig. 5f. Moreover, the specific capacitance of HPZC-4 decreases nearly linearly with current density increasing, which may be ascribed to electrode polarization (Fig. 5f). When the current density is increased to $10 \mathrm{~A} \mathrm{~g}^{-1}$, the specific capacitance of HPZC-4 is $3171 \mathrm{~F} \mathrm{~g}^{-1}$ with the capacitance retention rate of $53.1 \%$ compared with at $1 \mathrm{~A} \mathrm{~g}^{-1}$, indicating the advanced rate capability of HPZC.

The origin of capacitance in the hybrid materials was clarified by EIS Nyquist plots shown in Fig. 5g. Due to the existence of honeycomb-like pore in HPZC, constant phase elements (CPE) were used in the equivalent circuit model instead of ideal capacitors (Fig. 5i) [61]. During the electrochemical process, ions diffuse from the electrolyte to the surface of $\mathrm{g}-\mathrm{C}_{3} \mathrm{~N}_{4}$ (CPE1) and ZnNCN/C composites (CPE2). Thus, the Warburg impedance $\left(\mathrm{W}_{0}\right.$, which represents the ion diffusion resistance) and the equivalent series resistance $\left(R_{S}\right.$, which represents the combination of the electrolyte resistance, the internal electrode resistance and the interface resistance) are in series with $\mathrm{CPE} 1$ and CPE2 [62]. Due to the independent charge storage processes nature of pseudocapacitance, CPE1 and CPE2 are connected in parallel. Moreover, since the redox electrochemical reaction is controlled by the kinetics of charge transfer at the electrode-electrolyte interface, CPE2 is connected in series with the charge transfer resistor $\left(\mathrm{R}_{\mathrm{ct}}\right)$. As shown in Fig. $5 \mathrm{i}$, the equivalent circuit can properly describe the resistance and capacitance characteristics of ZnNCN based electrodes. According to the fitting results, HPZC4 has the lowest $\mathrm{R}_{\mathrm{S}}(1.234 \Omega)$, which is lower that of ZC $(1.449 \Omega)$ and ZnNCN (1.514 $\Omega$ ) (Fig. $5 \mathrm{~g}$ ). This may be attributed to the well-developed honeycomb-like pore structure of HPZC with significant quantity of heteroatoms on its surface resulting from $\mathrm{g}-\mathrm{C}_{3} \mathrm{~N}_{4}$ coating, which effectively decreases the interface resistance $[63,64]$. In the low frequency region, HPZC-4 shows the largest slope, corresponding to the lowest total impedance, indicating that HPZC-4 has best penetrability of the electrolyte ions compared to those in $\mathrm{ZC}$ and $\mathrm{ZnNCN}$, which can be ascribed to the unique honeycomb-like porous structure and dense 2D material $\mathrm{g}-\mathrm{C}_{3} \mathrm{~N}_{4}$ surface loading effectively reducing ion diffusion resistance, thereby improving ion diffusion efficiency [23,65]. Moreover, the fast ion diffusion is an important prerequisite for high specific capacitance and excellent rate performance, indicating the potential excellent electrochemical performance of HPZC electrodes.

The durable ability of the ZnNCN, ZC and HPZC-4 electrode materials was evaluated under 3,000 continuous deep charge-discharge cycles at $7 \mathrm{~A} \mathrm{~g}^{-1}$. As shown in Fig. 5h, the specific capacitance of ZNCN and $\mathrm{ZC}$ remains $67.1 \%$ and $81.3 \%$ respectively compared with the original capacitance. Moreover, HPZC-4 exhibits the highest initial capacitance $\left(3315 \mathrm{~F} \mathrm{~g}^{-1}\right.$ ) and show capacitance retention of $87.9 \%$ after 3,000 cycles with a decay rate of only $0.004 \%$ per cycle. These above results show 


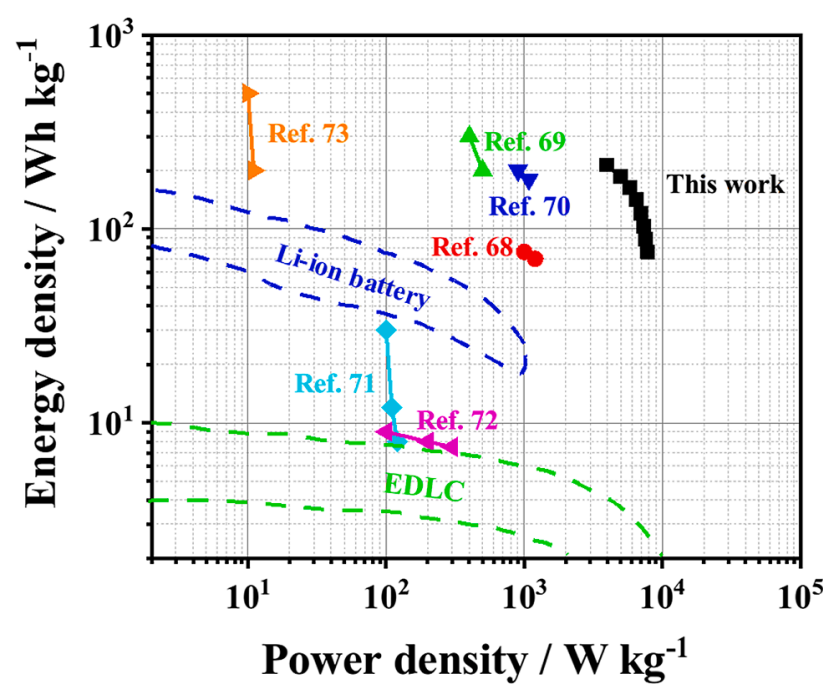

Fig. 7. Ragone plot of as-assembled HPZC-4//AC ASC and a comparison with the fields of EDLC, Li-ion batteries, and references.

that HPZC based supercapacitor has a solid structure and can work stably for a long time at high current density. In order to verify this hypothesis, we performed SEM analysis on the used HPZC-4 electrode after 3000 charge-discharge cycles. As shown in Fig. S8, it is clearly that the morphology of the HPZC-4 electrode after a long-term cycle test at high current density was nearly the same as that of the initial state. Although HPZC electrodes have made gratifying progress in cycle performance, however, the durable ability of HPZC-4 electrode still lower than some heteroatoms doped carbon materials[66], which may due to the Faraday-based oxidation-reduction reaction and poor conductivity of transition metal-based materials, resulting in the relatively poor cycle stability of pseudocapacitors composed of transition metal-based materials [67].

In order to measure the performance of HPZC electrode materials under real environment, an asymmetric supercapacitor (HPZC-4//AC
ASC) was assembled with HPZC-4 as the anode, activated carbon (AC) as the cathode, and $\mathrm{KOH}$ aqueous solution as the electrode solution. Moreover, the electrodes weight ratio was estimated to be 0.34 ( $\mathrm{m}$ (HPZC-4) / $m$ (AC)).

Fig. 6a shows typical CV curves for HPZC-4//AC ASC at various scan rates in the range of $0-1.6 \mathrm{~V}$. Obviously, the scan rate does not significantly affect the shape of CV curves, suggesting that HPZC-4//AC ASC can be used in related devices that require high operating rates and good reversibility. It is worth noting that an increase in a voltage setting higher than $1 \mathrm{~V}$ resulted in an unsatisfactory coulombic efficiency of HPZC-4//AC ASC of less than $90 \%$, thus a $0-1 \mathrm{~V}$ voltage range was chosen for further testing. As shown in Fig. S9, HPZC-4//AC ASC has an excellent coulombic efficiency ( $\geq 95 \%$ ) under various current densities (range from $3 \mathrm{~A} \mathrm{~g}^{-1}$ to $10 \mathrm{~A} \mathrm{~g}^{-1}$ ). Fig. $6 \mathrm{~b}$ shows the relevant GCD curves under the 3-10 $\mathrm{A} \mathrm{g}^{-1}$ and $0-1 \mathrm{~V}$ potential windows. According to the formula calculation, the specific capacitance of HPZC-4//AC ASC is 779 $\mathrm{F} \mathrm{g}^{-1}$ at $3 \mathrm{~A} \mathrm{~g}^{-1}, 765 \mathrm{~F} \mathrm{~g}^{-1}$ at $5 \mathrm{~A} \mathrm{~g}^{-1}$ and $720 \mathrm{~F} \mathrm{~g}^{-1}$ at $10 \mathrm{~A} \mathrm{~g}^{-1}$, respectively (Fig. 5d). Beides, when increasing the current density from $3 \mathrm{~A} \mathrm{~g}^{-1}$ to $10 \mathrm{~A} \mathrm{~g}^{-1}$, HPZC-4//AC ASC still shows $92.4 \%$ specific capacitance retention, indicating superior rate capability.

The cycle stability of the asymmetric supercapacitor HPZC-4//AC ASC is measured at $7 \mathrm{~A} \mathrm{~g}^{-1}$ for 2,000 deep charge-discharge cycles. As shown in Fig. 6c, after 2,000 cycles, HPZC-4//AC ASC can retain $87.6 \%$ specific capacitance compared with the initial capacitance value, corresponding to the average decay rate of $0.006 \%$ per cycle, and simultaneously provides high coulombic efficiency during these processes, which indicates the high cycle stability of HPZC-4//AC ASC. Furthermore, the power density and energy density of the HPZC and other related electrode materials are shown in Fig. 7. HPZC-4//AC ASC can provide maximum power density and energy density of $7839 \mathrm{~W}$ $\mathrm{Kg}^{-1}$ and $213 \mathrm{Wh} \mathrm{Kg}^{-1}$, respectively, which is obviously superior than many related reported literatures [68-73]. Moreover, connecting two HPZC-4//AC ASC cells in series can power the red-light emitting diode for at least $3 \mathrm{~min}$ (Fig. 8), which further shows that the HPZC based supercapacitor has high potential in practical applications.
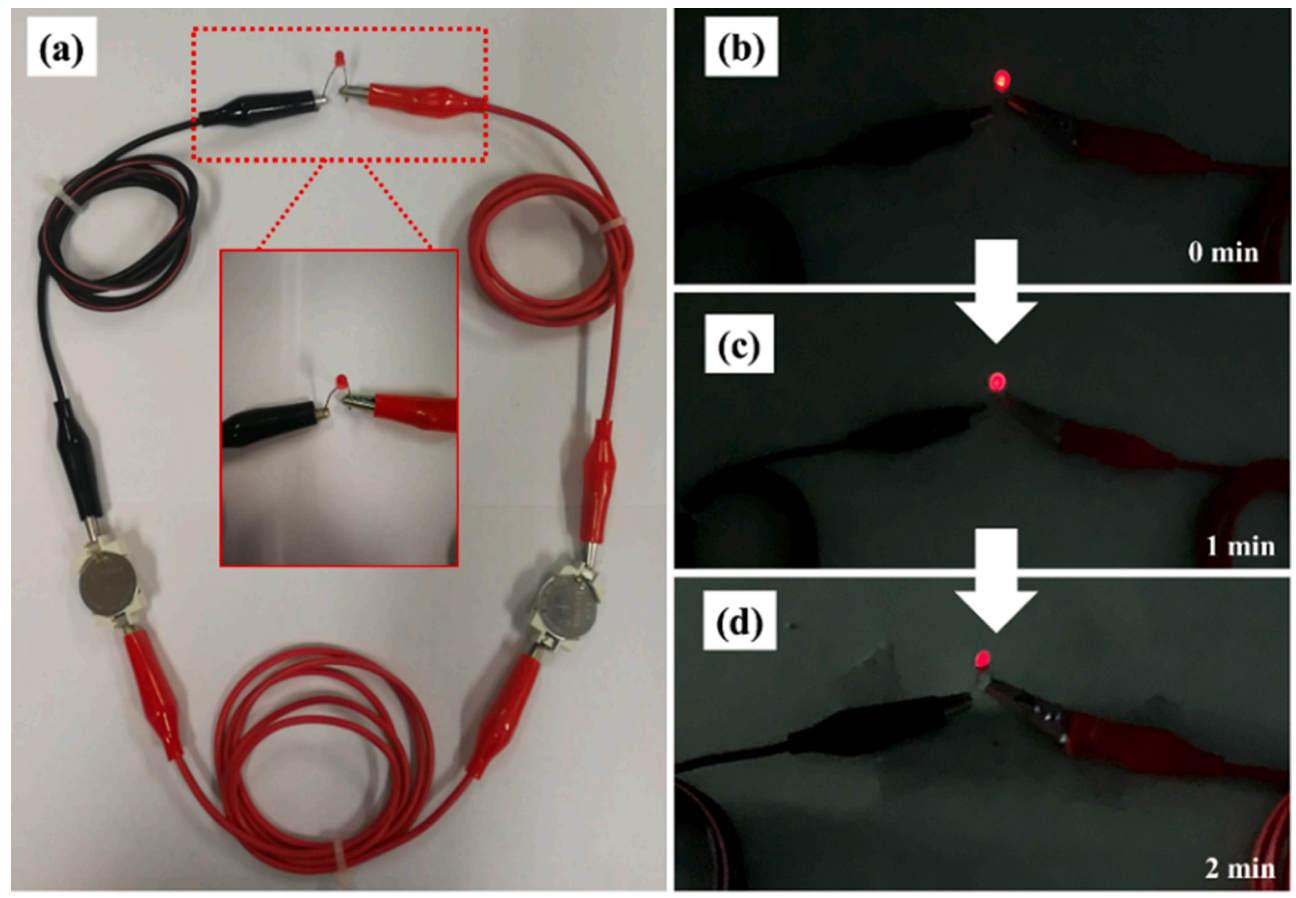

Fig. 8. (a) Red LED light powered by two HPZC-4//AC ASCs connected in series, and (b-d) corresponding the images of lighting time from 0 to 2 min. (For interpretation of the references to color in this figure legend, the reader is referred to the web version of this article.) 


\section{Conclusion}

Honeycomb-like porous $\mathrm{ZnNCN} / \mathrm{g}-\mathrm{C}_{3} \mathrm{~N}_{4}$ nanocomposites (HPZC) prepared using TRD-ZIF-8 as a precursor via a simple heat treatment method and suitable for the application as supercapacitor electrode materials were reported for the first time in this study. Optimized HPZC4 electrode demonstrated a specific capacitance of $5975 \mathrm{~F} \mathrm{~g}^{-1}$ at $1 \mathrm{~A} \mathrm{~g}^{-1}$ in a three-electrode testing system, significantly higher than those of reference materials ZnNCN and ZC. HPZC-4//AC ASC supercapacitor testing cell was assembled and displayed maximum power and energy densities of $7839 \mathrm{~W} \mathrm{Kg}^{-1}$ and $213 \mathrm{Wh} \mathrm{Kg}^{-1}$, respectively. The promising electrochemical performance of HPZC materials resulted from their well-developed honeycomb-like porous structure with g- $\mathrm{C}_{3} \mathrm{~N}_{4}$ surface loading responsible for the enhancement of surface area and conductivity and the reduction of ion diffusion resistance. This study provides a facile method to manufacture inexpensive transition metal carbodiimide materials as promising electrodes for asymmetric electrochemical capacitors.

\section{CRediT authorship contribution statement}

Jiaqi Shen: Investigation, Software. Xiaozhen Chen: Investigation, Validation, Software. Peng Wang: Supervision, Funding acquisition, Conceptualization, Writing - original draft. Feng Zhou: Software, Validation, Formal analysis. Lei Lu: Validation, Software. Rongfang Wang: Funding acquisition. Vladimir Linkov: Writing - review \& editing. Shan Ji: Supervision, Project administration, Funding acquisition.

\section{Declaration of Competing Interest}

The authors declare that they have no known competing financial interests or personal relationships that could have appeared to influence the work reported in this paper.

\section{Acknowledgements}

We would like to thank the financial support from the Zhejiang Provincial Natural Science Foundation of China (LQ19F040005), National Natural Science Foundation of China (51661008 and 21766032), Jiaxing Public Welfare Research Program (2018AY11007) and Jiaxing University SRT project (CD8517193136).

\section{Appendix A. Supplementary data}

Supplementary data to this article can be found online at https://doi. org/10.1016/j.apsusc.2020.148355.

\section{References}

[1] H.Y. Lee, J.B. Goodenough, Supercapacitor Behavior with KCl Electrolyte, J. Solid State Chem. 144 (1999) 220-223.

[2] L.L. Zhang, X.S. Zhao, Carbon-based materials as supercapacitor electrodes, Chem. Soc. Rev. 38 (2009) 2520-2531.

[3] L. Hou, R. Bao, Z. Chen, M. Rehan, L. Tong, G. Pang, C. Yuan, Comparative investigation of hollow mesoporous NiCo2S4 ellipsoids with enhanced pseudocapacitances towards high-performance asymmetric supercapacitors, Electrochim. Acta 214 (2016) 76-84.

[4] F. Liu, D. Xue, An Electrochemical Route to Quantitative Oxidation of Graphene Frameworks with Controllable C/O Ratios and Added Pseudocapacitances, Chem. Eur. J. 19 (2013) 10716-10722.

[5] W. Ni, B. Wang, J. Cheng, X. Li, Q. Guan, G. Gu, L. Huang, Hierarchical foam of exposed ultrathin nickel nanosheets supported on chainlike Ni-nanowires and the derivative chalcogenide for enhanced pseudocapacitance, Nanoscale 6 (2014) 2618-2623.

[6] S. Wang, J. Pu, Y. Tong, Y. Cheng, Y. Gao, Z. Wang, ZnCo2O4 nanowire arrays grown on nickel foam for high-performance pseudocapacitors, J. Mater. Chem. A 2 (2014) 5434-5440.

[7] Y. Pang, S. Zhang, S. Chen, J. Liang, M. Li, D. Ding, S. Ding, Transition-Metal Oxides Anchored on Nitrogen-Enriched Carbon Ribbons for High-Performance Pseudocapacitors, Chem. Eur. J. 24 (2018) 16104-16112.
[8] Y. Ji, C. Zhou, F. Lin, B. Li, F. Yang, H. Zhu, J. Duan, Z. Chen, Submicron-Sized NbDoped Lithium Garnet for High Ionic Conductivity Solid Electrolyte and Performance of Quasi-Solid-State Lithium Battery, Materials 13 (2020) 560.

[9] Y. Wang, J. Guo, T. Wang, J. Shao, D. Wang, Y.-W. Yang, Mesoporous Transition Metal Oxides for Supercapacitors, Nanomaterials 5 (2015) 1667-1689.

[10] A. Ehsani, A.A. Heidari, H.M. Shiri, Electrochemical Pseudocapacitors Based on Ternary Nanocomposite of Conductive Polymer/Graphene/Metal Oxide: An Introduction and Review to it in Recent Studies, Chem. Rec. 19 (2019) 908-926.

[11] M. Krott, X. Liu, B.P.T. Fokwa, M. Speldrich, H. Lueken, R. Dronskowski, Synthesis, Crystal-Structure Determination and Magnetic Properties of Two New TransitionMetal Carbodiimides: CoNCN and NiNCN, Inorg. Chem. 46 (2007) 2204-2207.

[12] A. Eguia-Barrio, E. Castillo-Martínez, F. Klein, R. Pinedo, L. Lezama, J. Janek, P. Adelhelm, T. Rojo, Electrochemical performance of CuNCN for sodium ion batteries and comparison with ZnNCN and lithium ion batteries, J. Power Sources 367 (2017) 130-137.

[13] Y. Xie, Y. Zhuo, S. Liu, Y. Lin, D. Zuo, X. Wu, C. Li, P.K. Wong, Ternary g-C3N4/ ZnNCN@ZIF-8 Hybrid Photocatalysts with Robust Interfacial Interactions and Enhanced CO2 Reduction Performance, Sol. RRL 4 (2020) 1900440.

[14] W. Zhao, J. Pan, F. Huang, Nonaqueous synthesis of metal cyanamide semiconductor nanocrystals for photocatalytic water oxidation, Chem. Commun. 54 (2018) 1575-1578.

[15] G. Wang, L. Zhang, J. Zhang, A review of electrode materials for electrochemical supercapacitors, Chem. Soc. Rev. 41 (2012) 797-828.

[16] S. Zhong, H. Liu, D. Wei, J. Hu, H. Zhang, H. Hou, M. Peng, G. Zhang, H. Duan, Long-aspect-ratio N-rich carbon nanotubes as anode material for sodium and lithium ion batteries, Chem. Eng. J. 395 (2020) 125054.

[17] G. Zhang, Y. Song, H. Zhang, J. Xu, H. Duan, J. Liu, Radially Aligned Porous Carbon Nanotube Arrays on Carbon Fibers: A Hierarchical 3D Carbon Nanostructure for High-Performance Capacitive Energy Storage, Adv. Funct. Mater. 26 (2016) 3012-3020.

[18] K. Poonam, A. Sharma, S.K. Arora, Tripathi, Review of supercapacitors: Materials and devices, J. Energy Storage 21 (2019) 801-825.

[19] A. González, E. Goikolea, J.A. Barrena, R. Mysyk, Review on supercapacitors: Technologies and materials, Renew. Sust. Energ. Rev. 58 (2016) 1189-1206.

[20] F. Grote, H. Zhao, Y. Lei, Self-supported carbon coated TiN nanotube arrays: innovative carbon coating leads to an improved cycling ability for supercapacitor applications, J. Mater. Chem. A 3 (2015) 3465-3470.

[21] K. Zou, Y. Deng, J. Chen, Y. Qian, Y. Yang, Y. Li, G. Chen, Hierarchically porous nitrogen-doped carbon derived from the activation of agriculture waste by potassium hydroxide and urea for high-performance supercapacitors, J. Power Sources 378 (2018) 579-588.

[22] L. Ma, H. Fan, K. Fu, S. Lei, Q. Hu, H. Huang, G. He, Protonation of Graphitic Carbon Nitride (g-C3N4) for an Electrostatically Self-Assembling Carbon@g-C3N4 Core-Shell Nanostructure toward High Hydrogen Evolution, ACS Sustain. Chem. Eng. 5 (2017) 7093-7103.

[23] B. Dong, M. Li, S. Chen, D. Ding, W. Wei, G. Gao, S. Ding, Formation of g-C3N4@Ni (OH)2 Honeycomb Nanostructure and Asymmetric Supercapacitor with High Energy and Power Density, ACS Appl. Mater. Interfaces 9 (2017) 17890-17896.

[24] M. Tahir, C. Cao, N. Mahmood, F.K. Butt, A. Mahmood, F. Idrees, S. Hussain, M. Tanveer, Z. Ali, I. Aslam, Multifunctional g-C3N4 Nanofibers: A Template-Free Fabrication and Enhanced Optical, Electrochemical, and Photocatalyst Properties, ACS Appl. Mater. Interfaces 6 (2014) 1258-1265.

[25] S.C. Yan, Z.S. Li, Z.G. Zou, Photodegradation Performance of g-C3N4 Fabricated by Directly Heating Melamine, Langmuir 25 (2009) 10397-10401.

[26] J. Fu, J. Yu, C. Jiang, B. Cheng, g-C3N4-Based Heterostructured Photocatalysts, Adv. Energy Mater. 8 (2018) 1701503.

[27] F. Dong, Z. Zhao, T. Xiong, Z. Ni, W. Zhang, Y. Sun, W.-K. Ho, In Situ Construction of g-C3N4/g-C3N4 Metal-Free Heterojunction for Enhanced Visible-Light Photocatalysis, ACS Appl. Mater. Interfaces 5 (2013) 11392-11401.

[28] B. Palanivel, S.d. Mudisoodum perumal, T. Maiyalagan, V. Jayarman, C. Ayyappan, M. Alagiri, Rational design of $\mathrm{ZnFe2O4/g-C3N4} \mathrm{nanocomposite} \mathrm{for} \mathrm{enhanced}$ photo-Fenton reaction and supercapacitor performance, Appl. Surf. Sci., 498 (2019) 143807.

[29] Z. Ou, X. Song, W. Huang, X. Jiang, S. Qu, Q. Wang, P.V. Braun, J.S. Moore, X. Li, Q. Chen, Colloidal Metal-Organic Framework Hexapods Prepared from Postsynthesis Etching with Enhanced Catalytic Activity and Rollable Packing, ACS Appl. Mater. Interfaces 10 (2018) 40990-40995.

[30] J. Troyano, A. Carné-Sánchez, C. Avci, I. Imaz, D. Maspoch, Colloidal metal-organic framework particles: the pioneering case of ZIF-8, Chem. Soc. Rev. 48 (2019) 5534-5546.

[31] Z. Tang, G. Zhang, H. Zhang, L. Wang, H. Shi, D. Wei, H. Duan, MOF-derived Ndoped carbon bubbles on carbon tube arrays for flexible high-rate supercapacitors, Energy Storage Mater. 10 (2018) 75-84.

[32] L. Wang, H. Liu, J. Zhao, X. Zhang, C. Zhang, G. Zhang, Q. Liu, H. Duan, Enhancement of charge transport in porous carbon nanofiber networks via ZIF-8enabled welding for flexible supercapacitors, Chem. Eng. J. 382 (2020) 122979.

[33] C. Avci, I. Imaz, A. Carné-Sánchez, J.A. Pariente, N. Tasios, J. Pérez-Carvajal, M. I. Alonso, A. Blanco, M. Dijkstra, C. López, D. Maspoch, Self-assembly of polyhedral metal-organic framework particles into three-dimensional ordered superstructures, Nat. Chem. 10 (2018) 78-84.

[34] Q. Li, D. Xu, J. Guo, X. Ou, F. Yan, Protonated g-C3N4@polypyrrole derived Ndoped porous carbon for supercapacitors and oxygen electrocatalysis, Carbon 124 (2017) 599-610.

[35] D. Wang, Y. Wang, Y. Chen, W. Liu, H. Wang, P. Zhao, Y. Li, J. Zhang, Y. Dong, S. Hu, J. Yang, Coal tar pitch derived N-doped porous carbon nanosheets by the in- 
situ formed g-C3N4 as a template for supercapacitor electrodes, Electrochim. Acta 283 (2018) 132-140.

[36] A.Y. Chen, T.T. Zhang, Y.J. Qiu, D. Wang, P. Wang, H.J. Li, Y. Li, J.H. Yang, X. Y. Wang, X.F. Xie, Construction of nanoporous gold/g-C3N4 heterostructure for electrochemical supercapacitor, Electrochim. Acta 294 (2019) 260-267.

[37] P. Matheswaran, P. Karuppiah, S.-M. Chen, P. Thangavelu, B. Ganapathi, Fabrication of g-C3N4 Nanomesh-Anchored Amorphous NiCoP2O7: Tuned Cycling Life and the Dynamic Behavior of a Hybrid Capacitor, ACS Omega 3 (2018) 18694-18704.

[38] J.J. Arayamparambil, M. Mann, X. Liu, M. Alfredsson, R. Dronskowski, L. Stievano, M.T. Sougrati, Electrochemical Evaluation of $\mathrm{Pb}, \mathrm{Ag}$, and $\mathrm{Zn}$ Cyanamides/ Carbodiimides, ACS Omega 4 (2019) 4339-4347.

[39] X. Li, M. Li, J. Yang, X. Li, T. Hu, J. Wang, Y. Sui, X. Wu, L. Kong, Synergistic effect of efficient adsorption g-C3N4/ZnO composite for photocatalytic property, J. Phys. Chem. Solids 75 (2014) 441-446.

[40] J.J. Arayamparambil, K. Chen, A. Iadecola, M. Mann, X. Qiao, B. Fraisse, R. Dronskowski, L. Stievano, M.T. Sougrati, Reversible High Capacity and Reaction Mechanism of Cr2(NCN)3 Negative Electrodes for Li-Ion Batteries, Energy Technol. 8 (2020) 1901260.

[41] R.J. Müller, J. Lan, K. Lienau, R. Moré, C.A. Triana, M. Iannuzzi, G.R. Patzke, Monitoring surface transformations of metal carbodiimide water oxidation catalysts by operando XAS and Raman spectroscopy, Dalton Trans. 47 (2018) 10759-10766.

[42] W. Guo, J. Wang, C. Fan, Z. Chen, P. Liu, D. Zhu, Z. Xu, L. Pang, T. Li, Synthesis of Carbon Self-Repairing Porous g-C3N4 Nanosheets/NiCo2S4 Nanoparticles Hybrid Composite as High-Performance Electrode Materials for Supercapacitors, Electrochim. Acta 253 (2017) 68-77.

[43] L. Zhao, X. Kuang, Z. Liu, Y. Hou, Z. Wang, Q. Wei, J.Y. Lee, B. Kang, Anchoring CuO Nanoparticles On C, N-Codoped G-C3N4 Nanosheets from MelamineEntrapped MOF Gel for High-Efficiency Oxygen Evolution, ChemNanoMat 5 (2019) 1170-1175.

[44] Y. Gong, X. Zhao, H. Zhang, B. Yang, K. Xiao, T. Guo, J. Zhang, H. Shao, Y. Wang, G. Yu, MOF-derived nitrogen doped carbon modified g-C3N4 heterostructure composite with enhanced photocatalytic activity for bisphenol A degradation with peroxymonosulfate under visible light irradiation, Appl. Catal. B 233 (2018) $35-45$.

[45] V. Nallathambi, N. Leonard, W. Patterson, K. Artyushkova, P. Atanassov, S. Calabrese Barton, Ammonia-Generating Precursors in MNC Electrocatalysts for Oxygen Reduction, Meeting Abstracts, MA2011-02 (2011) 1150.

[46] P.I.L. Hagouel, A.R. Neureuther, A.M. Zenk, Negative resist corner rounding, Envelope volume modeling, Journal of Vacuum Science \& Technology B: Microelectronics and Nanometer Structures Processing, Measurement, and Phenomena 14 (1996) 4257-4261.

[47] C. Avci, Y. Liu, J.A. Pariente, A. Blanco, C. Lopez, I. Imaz, D. Maspoch, TemplateFree, Surfactant-Mediated Orientation of Self-Assembled Supercrystals of MetalOrganic Framework Particles, Small 15 (2019) 1902520.

[48] J. Wen, J. Xie, X. Chen, X. Li, A review on g-C3N4-based photocatalysts, Appl. Surf. Sci. 391 (2017) 72-123.

[49] J. Fu, B. Zhu, C. Jiang, B. Cheng, W. You, J. Yu, Hierarchical Porous O-Doped gC3N4 with Enhanced Photocatalytic CO2 Reduction Activity, Small 13 (2017) 1603938.

[50] L. Cui, X. Ding, Y. Wang, H. Shi, L. Huang, Y. Zuo, S. Kang, Facile preparation of Zscheme WO3/g-C3N4 composite photocatalyst with enhanced photocatalytic performance under visible light, Appl. Surf. Sci. 391 (2017) 202-210.

[51] I. Papailias, T. Giannakopoulou, N. Todorova, D. Demotikali, T. Vaimakis, C. Trapalis, Effect of processing temperature on structure and photocatalytic properties of g-C3N4, Appl. Surf. Sci. 358 (2015) 278-286.

[52] M. Groenewolt, M. Antonietti, Synthesis of g-C3N4 Nanoparticles in Mesoporous Silica Host Matrices, Adv. Mater. 17 (2005) 1789-1792.

[53] X. Yan, Z. Ye, G. Ning, J. Li, B. Kang, Y. Wang, Molten salt pyrolysis synthesis of magnetic FeNCN nanorods and their visible-light-driven photocatalytic properties, Appl. Surf. Sci. 506 (2020) 145010.
[54] M. Samadi, H.A. Shivaee, M. Zanetti, A. Pourjavadi, A. Moshfegh, Visible light photocatalytic activity of novel MWCNT-doped ZnO electrospun nanofibers, J. Mol. Catal. A: Chem. 359 (2012) 42-48.

[55] B. Dong, M. Li, S. Chen, D. Ding, W. Wei, G. Gao, S. Ding, Formation of g-C3N4@ $\mathrm{Ni}(\mathrm{OH}) 2$ honeycomb nanostructure and asymmetric supercapacitor with high energy and power density, ACS Appl. Mater. Interfaces 9 (2017) 17890-17896.

[56] J. Xu, Y. Sun, M. Lu, L. Wang, J. Zhang, J. Qian, X. Liu, Fabrication of hierarchical MnMoO4·H2O@MnO2 core-shell nanosheet arrays on nickel foam as an advanced electrode for asymmetric supercapacitors, Chem. Eng. J. 334 (2018) 1466-1476.

[57] J. Xu, Y. Sun, M. Lu, L. Wang, J. Zhang, J. Qian, E.J. Kim, Fabrication of porous $\mathrm{Mn} 2 \mathrm{O} 3$ microsheet arrays on nickel foam as high-rate electrodes for supercapacitors, J. Alloy. Compd. 717 (2017) 108-115.

[58] C. Liu, C. Zhang, H. Fu, X. Nan, G. Cao, Exploiting High-Performance Anode through Tuning the Character of Chemical Bonds for Li-Ion Batteries and Capacitors, Adv. Energy Mater. 7 (2017) 1601127.

[59] R. Wang, S. Wang, X. Peng, Y. Zhang, D. Jin, P.K. Chu, L. Zhang, Elucidating the Intercalation Pseudocapacitance Mechanism of MoS2-Carbon Monolayer Interoverlapped Superstructure: Toward High-Performance Sodium-Ion-Based Hybrid Supercapacitor, ACS Appl. Mater. Interfaces 9 (2017) 32745-32755.

[60] S. Fleischmann, J.B. Mitchell, R. Wang, C. Zhan, D.-E. Jiang, V. Presser, V. Augustyn, Pseudocapacitance: From Fundamental Understanding to High Power Energy Storage Materials, Chem. Rev. 120 (2020) 6738-6782.

[61] J. Kang, J. Wen, S.H. Jayaram, A. Yu, X. Wang, Development of an equivalent circuit model for electrochemical double layer capacitors (EDLCs) with distinct electrolytes, Electrochim. Acta 115 (2014) 587-598.

[62] J. Li, W. Zhang, X. Zhang, L. Huo, J. Liang, L. Wu, Y. Liu, J. Gao, H. Pang, H. Xue, Copolymer derived micro/meso-porous carbon nanofibers with vacancy-type defects for high-performance supercapacitors, J. Mater. Chem. A 8 (2020) 2463-2471.

[63] Z. Zhou, T. Liu, A.U. Khan, G. Liu, Block copolymer-based porous carbon fibers, Sci. Adv. 5 (2019) eaau6852.

[64] T. Lin, I.-W. Chen, F. Liu, C. Yang, H. Bi, F. Xu, F. Huang, Nitrogen-doped mesoporous carbon of extraordinary capacitance for electrochemical energy storage, Science 350 (2015) 1508-1513.

[65] J.-G. Lee, J.-Y. Kim, S.-H. Kim, Effects of microporosity on the specific capacitance of polyacrylonitrile-based activated carbon fiber, J. Power Sources 160 (2006) 1495-1500.

[66] T. Liu, F. Zhang, Y. Song, Y. Li, Revitalizing carbon supercapacitor electrodes with hierarchical porous structures, J. Mater. Chem. A 5 (2017) 17705-17733.

[67] Y. Ouyang, X. Xia, H. Ye, L. Wang, X. Jiao, W. Lei, Q. Hao, Three-Dimensional Hierarchical Structure ZnO@C@NiO on Carbon Cloth for Asymmetric Supercapacitor with Enhanced Cycle Stability, ACS Appl. Mater. Interfaces 10 (2018) 3549-3561.

[68] B. Qu, Y. Chen, M. Zhang, L. Hu, D. Lei, B. Lu, Q. Li, Y. Wang, L. Chen, T. Wang, $\beta$-Cobalt sulfide nanoparticles decorated graphene composite electrodes for high capacity and power supercapacitors, Nanoscale 4 (2012) 7810-7816.

[69] F. Zhang, T. Zhang, X. Yang, L. Zhang, K. Leng, Y. Huang, Y. Chen, A highperformance supercapacitor-battery hybrid energy storage device based on graphene-enhanced electrode materials with ultrahigh energy density, Energ. Environ. Sci 6 (2013) 1623-1632.

[70] B. Li, F. Dai, Q. Xiao, L. Yang, J. Shen, C. Zhang, M. Cai, Nitrogen-doped activated carbon for a high energy hybrid supercapacitor, Energ. Environ. Sci 9 (2016) $102-106$.

[71] M. Gong, Y. Li, H. Zhang, B. Zhang, W. Zhou, J. Feng, H. Wang, Y. Liang, Z. Fan, J. Liu, H. Dai, Ultrafast high-capacity NiZn battery with NiAlCo-layered double hydroxide, Energ. Environ. Sci 7 (2014) 2025-2032.

[72] L.-F. Chen, Z.-H. Huang, H.-W. Liang, H.-L. Gao, S.-H. Yu, Three-Dimensional Heteroatom-Doped Carbon Nanofiber Networks Derived from Bacterial Cellulose for Supercapacitors, Adv. Funct. Mater. 24 (2014) 5104-5111.

[73] J. Zhao, H. Lai, Z. Lyu, Y. Jiang, K. Xie, X. Wang, Q. Wu, L. Yang, Z. Jin, Y. Ma J. Liu, Z. Hu, Hydrophilic Hierarchical Nitrogen-Doped Carbon Nanocages for Ultrahigh Supercapacitive Performance, Adv. Mater. 27 (2015) 3541-3545. 